\title{
Hardy-Sobolev Spaces Decomposition In Signal Analysis
}

\author{
Pei Dang*, Tao Qian†, Zhong You
}

\begin{abstract}
Some fundamental formulas and relations in signal analysis are based on amplitude-phase representations $s(t)=A(t) e^{i \varphi(t)}$ and $\hat{s}(\omega)=B(\omega) e^{i \psi(\omega)}$, where the amplitude functions $A(t)$ and $B(\omega)$ and the phase functions $\varphi(t)$ and $\psi(\omega)$ are assumed to be differentiable. They include the amplitude-phase representations of the first and the second order means of the Fourier frequency and the time, and the equivalence relation between two forms of the covariance. A proof of the uncertainty principle is also based on the amplitude-phase representations. In general, however, signals of finite energy do not have differentiable amplitude-phase representations. The study presented in this paper extends the classical formulas and relations to general signals of finite energy. Under the formulation of phase and amplitude derivatives based on Hardy-Sobolev spaces decomposition the extended formulas reveal new features, and contribute to the foundations of time-frequency analysis. The established theory is based on the equivalent classes of the $L^{2}$ space but not on particular representations of the classes. We also give a proof of the uncertainty principle using the amplitude-phase representations defined through Hardy-Sobolev spaces decomposition.
\end{abstract}

Keywords: mean of frequency, mean of time, covariance, uncertainty principle, Hilbert transform, Hardy space, Hardy-Sobolev space, amplitude-phase representation of signal, instantaneous frequency

2000 Mathematical Subject Classification: 30H05, 42A50, 42A38

\section{Introduction}

The work presented in this paper was motivated by the recent study on relations between the Fourier frequency and the analytic frequency of signals ([4], [5], [6], [14], [10], [22]), and especially on relations between their respective positivities. For a signal $s$ of finite energy, positivity of its Fourier frequency addresses the property that $\hat{s}(\omega)=0$ if $\omega<0$, or, equivalently, $\hat{s} \chi_{-}=\hat{s}$, where $\hat{s}$ is the Fourier transform of $s$ and $\chi_{-}$is the characteristic (indicator) function of the set $(-\infty, 0)$. In contrast, positivity of analytic frequency amounts to require the analytic phase derivative $\varphi^{\prime}$ to be non-negative a.e., where $\phi$ is defined (if it could be) through $\rho(t) e^{i \varphi(t)}=s(t)+i H s(t)$ which is the so called analytic signal

\footnotetext{
*Pei Dang. Department of Mathematics, University of Macau, Macao (Via Hong Kong). Email: ya77408@umac.mo.

${ }^{\dagger}$ Tao Qian. Department of Mathematics, University of Macau, Macao (Via Hong Kong). Email: fsttq@umac.mo. The work was partially supported by Macao Sci. and Tech. Develop. Fund 051/2005/A. and NNSF of China (grant no.10771130)

${ }^{\ddagger}$ Zhong You. Faculty of Information Technology, Macau University of Science and Tecnology, Macao (Via Hong Kong). Email: zyou@must.edu.mo.
} 
associated to $s$, where $H$ stands for Hilbert transformation, defined by (see also the basic result 4 stated in $\S 2)$

$$
H s(t) \triangleq \frac{1}{\pi} \lim _{\epsilon \rightarrow 0} \int_{|t-u|>\epsilon} \frac{s(u)}{t-u} d u .
$$

The terminology analytic phase derivative will be often abbreviated as phase derivative. In this paper, when one side of an equality is defined through the other side of it, we write " $\triangleq$ " instead of " $="$.

The function $s+i H s$ is the boundary value of an analytic function in the upper-half complex plane, and thus possesses only positive Fourier frequencies (see the basic results 3 and 4 in $\S 2$ ). One thought that positivity of the Fourier frequency would imply positivity of analytic frequency. But it is not true. Outer functions in the upper-half complex plane (signals of minimum phase and minimum energy delay) are of positive Fourier frequencies, but they have negative phase derivatives on sets of positive Lebesgue measure ([18]). The wrong thought is caused by mixing up the two different kinds of frequencies. The result (1.2) given in Theorem 1.1, however, indicates that they indeed have some relations.

In the sequel we always assume the signal under study is of unit energy, that is

$$
\|s\|_{2}^{2}=\int_{-\infty}^{\infty}|s(t)|^{2} d t=1 .
$$

A precise version of a result given and essentially proved in [4] is formulated as follow.

Theorem 1.1 Let $s(t)=A(t) e^{i \varphi(t)} \in L^{2}(\mathbb{R}), A(t)=|s(t)|$. Assume that the classical derivatives $\frac{d A}{d t}, \frac{d \varphi}{d t}$ and $\frac{d s}{d t}$ all exist and are Lebesgue measurable, and $\frac{d s}{d t}$ is in $L^{2}(\mathbb{R})$. Then there holds

$$
\langle\omega\rangle=\int_{-\infty}^{\infty} \frac{d \varphi(t)}{d t}|s(t)|^{2} d t,
$$

where $\langle\omega\rangle$ is defined by

$$
\langle\omega\rangle \triangleq \int_{-\infty}^{\infty} \omega|\hat{s}(\omega)|^{2} d \omega
$$

The formula (1.2) shows that if the mean of the Fourier frequency is positive then the mean of the analytic frequency $\frac{d \varphi(t)}{d t}$ is also positive, and vice versa. If, in particular, $s$ is taken to be an analytic signal, that is, $s=s_{1}+i H s_{1}$ for some signal $s_{1} \in L^{2}(\mathbb{R})$, then $s$ is of positive Fourier frequency and the above formulas imply

$$
0<\langle\omega\rangle=\int_{0}^{\infty} \omega|\hat{s}(\omega)|^{2} d \omega=\int_{-\infty}^{\infty} \frac{d \varphi(t)}{d t}|s(t)|^{2} d t
$$

In such case, however, there does not necessarily hold $\frac{d \varphi(t)}{d t} \geq 0$, a.e.

For a real-valued signal $s_{1}$ and $s=s_{1}+i H s_{1}=\rho e^{i \varphi}$, if and only if the phase derivative $\frac{d \varphi}{d t} \geq 0$, a.e., then $\frac{d \varphi}{d t} \geq 0$ is called the analytic instantaneous frequency function of $s_{1}$, abbreviated as instantaneous frequency of $s_{1}$. We call a real-valued signal a mono-component if and only if its associated analytic signal possesses positive (non-negative) phase derivative ([17]). Note that phase derivative $\frac{d \varphi}{d t}$ should be suitably defined and the definition is given in $\S 3$. The positivity of analytic phase derivative and related issues have been the subject of much controversy. The positivity requirement for frequency 
has support from physics science. We now provide more background information for the interest in signals of positive analytic phase derivative in relation to time-frequency analysis.

As in Theorem 1.1, let $s(t)=A(t) e^{i \varphi(t)} \in L^{2}(\mathbb{R}), A(t)=|s(t)|$, and $\hat{s}(\omega)=B(\omega) e^{i \psi(\omega)}, B(\omega)=$ $|\hat{s}(\omega)|$. One usually assumes a time-frequency distribution $P(t, \omega)$ to satisfy the positivity condition

$$
P(t, \omega) \geq 0,
$$

and the usual edge distribution conditions

$$
P_{F}(\omega) \triangleq \int_{-\infty}^{\infty} P(t, \omega) d t=|\hat{s}(\omega)|^{2}, \quad P_{T}(t) \triangleq \int_{-\infty}^{\infty} P(t, \omega) d \omega=|s(t)|^{2} .
$$

One further expects to have

$$
\langle\omega\rangle_{t} \triangleq \frac{1}{P_{T}(t)} \int_{-\infty}^{\infty} \omega P(t, \omega) d \omega=\frac{d \varphi(t)}{d t},
$$

and

$$
\langle t\rangle_{\omega} \triangleq \frac{1}{P_{F}(\omega)} \int_{-\infty}^{\infty} t P(t, \omega) d t=-\frac{d \psi(\omega)}{d \omega} .
$$

The left-hand-side of (1.6) is the conditional mean of the Fourier frequency at the time moment $t$, while that of (1.7) is the conditional mean of the time at the frequency $\omega$. Wigner-Ville distribution, for instance, satisfies the conditions (1.5), (1.6) and (1.7), but not (1.4).

We also expect the analytic positivity property that

$$
\hat{s} \chi_{-}=0 \quad \text { implies } P(t, \cdot) \chi_{-}=0, \quad \forall t
$$

for the distribution. Wigner-Ville distribution, for instance, has this property. If (1.8) holds, then (1.4) and (1.6) imply $\frac{d \varphi(t)}{d t} \geq 0$. In other words, positivity of phase derivative is a necessary condition of positivity of Fourier frequency, positivity (1.4) and analytic positivity (1.8) and the relation for the conditional mean of Fourier frequencies (1.6) of the time-frequency distributions.

Positivity of phase derivative is crucial in order to define meaningful (analytic) instantaneous frequency (as a function). The observation on analytic outer functions tells that one cannot expect positivity of analytic phase derivative in general. What one could do would be to decompose signals of positive Fourier frequency into sums of mono-components ([17]). Fourier series is the classical example of such decomposition. Expansions in Takenaka-Malmquist systems as generalizations of Fourier series belong to the same category ([1], [3], [9]). Now comes the concept adaptive monocomponent decomposition where adaptivity is referred to fast convergence of the decomposition ([19], [20]). In a fast decomposition of a given signal intrinsic mono-components of the signal are extracted out. The present work is not to pursue this direction for which the reader is referred to ([17], [18], [19]).

To study positivity of phase derivative, we first need to define the notion phase derivative. The present paper is set to study the right forms (replacements) of fundamental formulas such as (1.2), (1.9) and (1.10 for the general cases where classical derivative $\frac{d \varphi}{d t}$ and $\frac{d \psi}{d t}$ may not exist. For all purposes the right definition of phase derivative is crucial. In general cases $e^{i \varphi(t)}$ is defined by $s /|s|$, but $\varphi(t)$ is not uniquely defined, let alone $\frac{d \varphi(t)}{d t}$. Moreover, $s$ may not be a smooth function, and when it is, then $H s$ may not, and $\varphi(t)$ may not be smooth. 
Similar queries arise from the study of signal of minimum phase and minimum energy delay in relation to all-pass filters (see [12],[13]). In the complex analysis terminology, signals of minimum phase and minimum energy delay are (analytic) outer functions and all-pass filters are (analytic) inner functions in the corresponding domains. The related results in signal analysis are only interplay relations between the two types of analytic functions. To the authors' knowledge, before ([18]), no literature has given a valid proof for the fact that outer functions are of minimum phase, or, equivalently, inner functions are of positive "phase derivatives" on the boundary, and no literature has defined what is the boundary phase derivative of a general $Z$-transform. Before [18], technicalities in the literature were only valid for proving the positivity of the phase derivative of finite Blaschke products and of singular inner functions induced by a finite linear combination of shifted Dirac point measures. In [18], existence and positivity of phase derivative of inner functions are reduced to the classical Julia-Wolff-Carathéodory Theorem, and existence and zero-mean property of a class of outer functions are proved.

In this paper, based on the definition of phase derivative and amplitude derivative of signals in the Hardy-Sobolev space extensions of (1.2) and of the following relations are given:

$$
\sigma_{\omega}^{2}=\int_{-\infty}^{\infty}\left(\frac{d A(t)}{d t}\right)^{2} d t+\int_{-\infty}^{\infty}\left(\frac{d \varphi(t)}{d t}-\langle\omega\rangle\right)^{2} A^{2}(t) d t
$$

and

$$
\operatorname{Cov}_{t \omega} \triangleq\left\langle t \frac{d \varphi(t)}{d t}\right\rangle-\langle t\rangle\langle\omega\rangle=-\left\langle\omega \frac{d \psi(\omega)}{d \omega}\right\rangle-\langle t\rangle\langle\omega\rangle
$$

where

$$
\left\langle t \frac{d \varphi(t)}{d t}\right\rangle \triangleq \int_{-\infty}^{\infty} t \frac{d \varphi(t)}{d t} A^{2}(t) d t, \quad \text { and } \quad\left\langle\omega \frac{d \psi(\omega)}{d \omega}\right\rangle \triangleq \int_{-\infty}^{\infty} \omega \frac{d \psi(\omega)}{d \omega} B^{2}(\omega) d \omega .
$$

These relations are formulated in time-frequency analysis with important applications. Our contribution in this paper is two-fold: (i) We generalize the concept "phase derivative", $\frac{d \varphi}{d t}$, to non-smooth square-integrable signals in the Sobolev space; and (ii) As application of this generalization, we show that the formulas $(1.2),(1.9),(1.10)$ are all extendable with new forms to general signals. We also give a proof of the classical uncertainty principle by using the generalized phase derivatives.

Throughout the paper we assume that $s$ is complex-valued. In applications we usually assume $s$ is real-valued, and the Hilbert transform $H s$ contributes the pure imaginary part to form the associated analytic signal $s+i H s$. The generalizations of the phase and amplitude derivatives are achieved via Hardy-Sobolev spaces decomposition of functions in the Sobolev space.

Throughout the paper we denote by $\mathbb{C}$ the complex plane, and by $\mathbb{C}^{+}$and $\mathbb{C}^{-}$the upper- and lower-half complex planes, respectively. Now we introduce the Hardy spaces in the upper- and the lower-half complex planes. For $1 \leq p<\infty$ the totality of the analytic functions in the upper-half complex plane $\mathbb{C}^{+}$under the norm

$$
\|s\|_{p} \triangleq \sup _{y>0}\left(\int_{-\infty}^{\infty}|s(t+i y)|^{p} d t\right)^{1 / p}<\infty
$$

forms a Banach space. For $0<p<1$, the totality of analytic functions in $\mathbb{C}^{+}$satisfying (1.12) under the distance

$$
d(s, u) \triangleq \sup _{y>0} \int_{-\infty}^{\infty}|s(t+i y)-u(t+i y)|^{p} d t<\infty
$$


forms a complete metric space. For $p=\infty$ the totality of the analytic functions in $\mathbb{C}^{+}$under the norm

$$
\|s\|_{\infty}=\sup \left\{|s(z)|: z \in \mathbb{C}^{+}\right\}<\infty
$$

forms a Banach space. In all the three cases we denote the space by $H^{p}\left(\mathbb{C}^{+}\right)$, and call it a Hardy space. Similarly one defines the Hardy spaces $H^{p}\left(\mathbb{C}^{-}\right)$for the lower-half complex plane.

Hardy spaces have a well developed theory connecting complex analysis and harmonic analysis. The latest developments of Hardy spaces mainly concern the real-Hardy spaces with the index region $0<p \leq 1$, and mainly via real analysis methods. For our purpose we concentrate in the complex Hardy spaces $H^{2}\left(\mathbb{C}^{ \pm}\right)$.

We adopt the notation for Sobolev spaces $L_{n}^{2}(\mathbb{R})$ in [23], that is

$$
L_{n}^{2}(\mathbb{R})=\left\{s(t) \in L^{2}(\mathbb{R}):\left(\frac{d^{*}}{d t}\right)^{n} s(t) \in L^{2}(\mathbb{R})\right\}
$$

with the norm defined by

$$
\sqrt{\|s\|_{2}^{2}+\left\|\left(\frac{d^{*}}{d t}\right)^{n} s\right\|_{2}^{2}},
$$

where $\left(\frac{d^{*}}{d t}\right)^{n} s(t)$ denotes the $n$-th distributional derivative of $s$.

Throughout this paper we will assume signals $s$ under study to satisfy

$$
s \in L^{2}(\mathbb{R}), \quad \omega \hat{s}(\omega) \in L^{2}(\mathbb{R}) .
$$

Signals satisfying the condition (1.13) belong to $L_{1}^{2}(\mathbb{R})$ (see Lemma 2.5).

If $s \in L^{2}(\mathbb{R})$, then we usually perform the Hardy spaces decomposition $s=s^{+}+s^{-}, s^{ \pm}=$ $(1 / 2)(s \pm i H s)$, and $s^{ \pm}$are, respectively, the boundary values of the analytic functions

$$
s^{ \pm}(z)=\frac{ \pm 1}{2 \pi i} \int_{-\infty}^{\infty} \frac{s(u)}{u-z} d u=\frac{1}{\sqrt{2 \pi}} \int_{-\infty}^{\infty} e^{i t \omega} \chi_{ \pm}(\omega) e^{-y \omega} \hat{s}(\omega) d \omega, \quad z=t+i y \in \mathbb{C}^{ \pm} .
$$

The last equal relation is a consequence of $(2.17)$, where $\chi_{ \pm}=\chi_{\mathbb{R}^{ \pm}}$, where $\mathbb{R}^{+}=[0,+\infty)$ and $\mathbb{R}^{-}=(-\infty, 0]$, and, in general, $\chi_{E}$ is the characteristic function of the Lebesgue measurable set $E$ that takes value 1 on $E$ and 0 otherwise. If $s$ satisfies the conditions in (1.13), that is $s \in L_{1}^{2}(\mathbb{R})$, then

$$
s^{ \pm} \in H^{ \pm} L_{1}^{2}(\mathbb{R})=\left\{s \in H^{2}\left(\mathbb{C}^{ \pm}\right): \frac{d^{*} s}{d t} \in H^{2}\left(\mathbb{C}^{ \pm}\right)\right\},
$$

where $H^{ \pm} L_{1}^{2}(\mathbb{R})$ are called Hardy-Sobolev spaces in, respectively, the upper- and lower-half complex planes. Thus, we have the decomposition

$$
L_{1}^{2}(\mathbb{R})=H^{+} L_{1}^{2}(\mathbb{R}) \bigoplus H^{-} L_{1}^{2}(\mathbb{R}) .
$$

The spaces $H^{+} L_{1}^{2}(\mathbb{R})$ and $H^{-} L_{1}^{2}(\mathbb{R})$ are mutually orthogonal. We note that in the function space notations we mix up the analytic functions in the respective domains with their boundary values due to the certain isometric isomorphism relationships in the respective contexts.

It is for the functions in the Hardy-Sobolev spaces that we are able to define phase and amplitude derivatives. They are defined to be non-tangential boundary limits of the same quantities in the respective domains in which the functions are analytic. The details will be given in $\S 3$. 
Both Hardy and Sobolev spaces have been well studied, and have long histories with ample applications. Hardy-Sobolev spaces in recent years have undergone a new phase of development ([2], [11]). To the authors' knowledge, no applications in signal analysis have been noted.

In $\S 2$ we discuss the useful relations between some five types of derivatives and in $\S 3$ we define the amplitude and the phase derivatives for $s^{+}$and $s^{-}$. In $\S 4$ we deal with the first and the second order means of the Fourier frequency. In $\S 5$ we give the results on the first and the second order means of the time. In $\S 6$ we study covariance, and in $\S 7$ we give a proof of the uncertainty principle that are all based on the generalized phase-amplitude representations. In $\S 8$ we draw the conclusions and give some remarks.

\section{Technical Preparations}

For any $\alpha>0$, define the $\alpha$-cone $\Gamma_{\alpha}^{+}(t)$ at $t \in \mathbb{R}$ by

$$
\Gamma_{\alpha}^{+}(t) \triangleq\{(x, y):|x-t|<\alpha y, 0<y<\infty\} .
$$

For an analytic function $s$ in $\mathbb{C}^{+}$define its $\alpha$-non-tangential maximal function by

$$
M_{\alpha}^{+} s(t) \triangleq \sup _{z \in \Gamma_{\alpha}^{+}(t)}|s(z)| .
$$

Then $M_{\alpha}^{+} s$ is a function defined on $\mathbb{R}$. It is a fundamental result that $M_{\alpha}^{+} s \in L^{2}(\mathbb{R})$ if and only if $s \in H^{2}\left(\mathbb{C}^{+}\right)([8])$. This result presents the equivalence between the two statements of which one is dependent of $\alpha$ but the other is not. This shows that the condition $M_{\alpha}^{+} s \in L^{2}(\mathbb{R})$ is independent of $\alpha$. In other words, if for any $\alpha \in(0, \infty)$ there holds $M_{\alpha}^{+} s \in L^{2}(\mathbb{R})$, then the latter relation holds for all $\alpha \in(0, \infty)$. One defines the $\alpha$-non-tangential maximal function for analytic functions in the lowerhalf complex plane $\mathbb{C}^{-}$in the same way through the $\alpha$-cone $\Gamma_{\alpha}^{-}(t)$ symmetric to $\Gamma_{\alpha}^{+}(t)$ with respect to the real-axis. The theory for the lower-half complex plane is parallel to that for the upper-half complex plane. We will write $\Gamma_{\alpha}(t)$ for either $\Gamma_{\alpha}^{+}(t)$ or $\Gamma_{\alpha}^{-}(t)$ depending on the context. To indicate a non-tangential limit $\sigma$ being independent of $\alpha>0$, we adopt the notation

$$
\lim _{\Gamma^{ \pm}: z \rightarrow t} s(z) \triangleq \sigma
$$

and this is exactly what happens to all $s \in H^{2}\left(\mathbb{C}^{ \pm}\right)$. Here we allow the limit $\sigma$ to be $\infty$, including $\pm \infty$. In the context this value $\sigma$ is denoted by $s(t)$. The correspondence between $s(z) \in H^{2}\left(\mathbb{C}^{ \pm}\right)$ and its boundary value $s(t) \in L^{2}(\mathbb{R})$ is one to one with equal norms in their respective spaces. For meromorphic functions in the respective domains, we use the same limit notation but for truncated $\alpha$-cones. Recall that a function is meromorphic in a domain if it is analytic throughout the domain except at its poles. This is exactly what is needed in defining phase and amplitude derivatives (see Theorem 3.1).

Now we list five types of derivatives. Assume that $s \in L^{2}(\mathbb{R})$.

(i) The distributional or weak derivative $\frac{d^{*} s}{d t}$. Based on the distribution theory this type of derivatives always exist.

(ii) The classical or strong derivative $\frac{d s}{d t}$. It may or may not exist.

(iii) The Fourier transform derivative that is the inverse Fourier transform of $i \omega \hat{s}(\omega)$ in $L^{2}(\mathbb{R})$. This will exist if $s \in L_{1}^{2}(\mathbb{R})$. 
(iv) Further assuming $s \in L_{1}^{2}(\mathbb{R})$, and decomposing $s$ into the sum $s=s^{+}+s^{-}$, where $s^{ \pm}$are the non-tangential boundary values of the associated analytic functions $s^{ \pm}(z)$ in the Hardy spaces $H^{2}\left(\mathbb{C}^{ \pm}\right)$. One can show that $s^{ \pm^{\prime}}(z)$ are also in $H^{2}\left(\mathbb{C}^{ \pm}\right)$, respectively, and therefore the non-tangential limits, called analytic derivatives,

$$
\lim _{\Gamma^{ \pm}: z \rightarrow t} s^{ \pm^{\prime}}(z) \triangleq s^{ \pm^{\prime}}(t), \quad z \in \mathbb{C}^{ \pm}
$$

exist.

(v) The boundary derivative defined through the non-tangential boundary limits

$$
\lim _{\Gamma^{ \pm}: z \rightarrow t} \frac{s^{ \pm}(z)-s^{ \pm}(t)}{z-t} \triangleq s^{ \pm^{\prime}}(t)
$$

provided that the non-tangential boundary values $s^{ \pm}(t)$ exist, and the non-tangential limits on the left-hand-side exist.

In order to define phase and amplitude derivatives for $s \in L_{1}^{2}(\mathbb{R})$ a close study on the relations between the five types of derivatives are necessary and that is what will be done in the rest of this section.

The Fourier transform of $s \in L^{1}(\mathbb{R})$ is defined by

$$
\hat{s}(\omega) \triangleq \frac{1}{\sqrt{2 \pi}} \int_{-\infty}^{\infty} e^{-i t \omega} s(t) d t .
$$

If $\hat{s}$ is also in $L^{1}(\mathbb{R})$, then the inversion formula holds, that is

$$
s(t)=\frac{1}{\sqrt{2 \pi}} \int_{-\infty}^{\infty} e^{i t \omega} \hat{s}(\omega) d \omega, \quad \text { a.e. }
$$

There holds the Plancherel Theorem

$$
\|\hat{s}\|_{2}^{2}=\|s\|_{2}^{2}, \quad s \in L^{1}(\mathbb{R}) \cap L^{2}(\mathbb{R}) .
$$

Through a density argument, both the Fourier transformation and its inverse can be extended to $L^{2}(\mathbb{R})$ in which Plancherel Theorem and the inversion remain to hold. When we use the formulas (2.14) and $(2.15)$ for $L^{2}(\mathbb{R})$ functions, we keep in mind that convergence of the integrals is taken to be in $L^{2}(\mathbb{R})$.

Now we summarize the results that are often recalled and regarded as basic results throughout the paper (see also[8], [21]).

1. $s$ belongs to $H^{2}\left(\mathbb{C}^{ \pm}\right)$if and only if $M_{\alpha}^{ \pm} s$ belongs to $L^{2}(\mathbb{R}), \alpha>0$. Such $s$ and $M_{\alpha}^{ \pm} s$ are of equivalent norms in their respective spaces.

2. If $s \in H^{2}\left(\mathbb{C}^{ \pm}\right)$, then the non-tangential boundary limit or non-tangential boundary value

$$
\lim _{\Gamma: z \rightarrow t} s(z)=s(t)
$$

exists for a.e. $t \in \mathbb{R}$. We will use the notation $s$ for both $s(z)$ and $s(t)$. They are of equal norms in their respective spaces. 
3. If $s \in L^{2}(\mathbb{R})$, then $s=s^{+}+s^{-}, \hat{s^{ \pm}} \triangleq \chi_{ \pm} \hat{s}, s^{ \pm} \in H^{2}\left(\mathbb{C}^{ \pm}\right)$, where

$$
s^{ \pm}(t)=\frac{ \pm 1}{\sqrt{2 \pi}} \int_{0}^{ \pm \infty} e^{i t \omega} \hat{s}(\omega) d \omega \text { and } s^{ \pm}(z)=\frac{ \pm 1}{2 \pi i} \int_{-\infty}^{\infty} \frac{s(u)}{u-z} d u, \quad z \in \mathbb{C}^{ \pm} .
$$

The decomposition is orthogonal and unique.

4. For $s \in L^{2}(\mathbb{R})$ we have the Plemelj Theorem

$$
\lim _{y \rightarrow \pm 0} \frac{1}{2 \pi i} \int_{-\infty}^{\infty} \frac{s(u)}{u-(t+i y)} d u=\frac{1}{2} s(t) \pm \frac{i}{2} H s(t)=s^{ \pm}(t), \quad \text { a.e. },
$$

where $H s$ is the Hilbert transform of $s$,

$$
H s(t)=\frac{1}{\pi} \text { p.v. } \int_{-\infty}^{\infty} \frac{s(u)}{t-u} d u=\frac{-i}{\sqrt{2 \pi}} \int_{-\infty}^{\infty} e^{i t \omega} \operatorname{sgn}(\omega) \hat{s}(\omega) d \omega,
$$

where p.v. stands for the principal value of the integral defined through (1.1) and $\operatorname{sgn}(\omega)$ is the signum function that takes value 1 for $\omega>0$ and value -1 for $\omega<0$.

5.

$$
\left.H^{2}=-I \text { ( } I \text { being the identity operator }\right), \quad\|H s\|_{L^{2}(\mathbb{R})}=\|s\|_{L^{2}(\mathbb{R})},
$$

and the adjoint operator of $H$ is $-H$.

6. The following Fourier transform plays an important role: For $z=x+i y, \pm y>0$,

$$
\left[\frac{1}{(\cdot)-z}\right]^{\vee}(\omega)= \pm \sqrt{2 \pi} i \chi_{ \pm}(\omega) e^{i x \omega} e^{-y \omega}
$$

The following result shows that, in the Hardy spaces, existence of the analytic derivative implies existence of the Fourier transform derivatives of the same and lower orders, and vice versa.

Lemma 2.1 Let $s \in H^{2}\left(\mathbb{C}^{ \pm}\right)$and $n$ a positive integer. Then

(i) $s^{(n)} \in H^{2}\left(\mathbb{C}^{ \pm}\right)$if and only if $\omega^{n} \hat{s} \in L^{2}(\mathbb{R})$.

(ii) $s^{(n)} \in H^{2}\left(\mathbb{C}^{ \pm}\right)$implies $s^{(k)} \in H^{2}\left(\mathbb{C}^{ \pm}\right), k=1, \ldots, n-1$.

Proof of Lemma We only prove the lemma for $H^{2}\left(\mathbb{C}^{+}\right)$. For $H^{2}\left(\mathbb{C}^{-}\right)$the proof is similar.

(i) For $s \in H^{2}\left(\mathbb{C}^{+}\right)$, denote $s_{y}(t)=s(t+i y)$. By invoking the basic results 3 and 6 , and the Plancherel Theorem, we have

$$
\begin{aligned}
s_{y}(t) & =\frac{1}{2 \pi i} \int_{-\infty}^{\infty} \frac{s(u)}{u-(t+i y)} d u \\
& =\frac{1}{\sqrt{2 \pi}} \int_{0}^{\infty} e^{i t \omega} e^{-y \omega} \hat{s}(\omega) d \omega .
\end{aligned}
$$

Therefore,

$$
\left(s_{y}\right)^{\wedge}(\omega)=\chi_{+}(\omega) e^{-y \omega} \hat{s}(\omega) .
$$


As consequence,

$$
\left(s_{y}^{(n)}\right)^{\wedge}(\omega)=(i \omega)^{n} \chi_{+}(\omega) e^{-y \omega} \hat{s}(\omega) .
$$

By the Plancherel Theorem,

$$
\int_{-\infty}^{\infty}\left|s^{(n)}(t+i y)\right|^{2} d t=\int_{0}^{\infty} \omega^{2 n} e^{-2 y \omega}|\hat{s}(\omega)|^{2} d \omega .
$$

Therefore, $s^{(n)} \in H^{2}\left(\mathbb{C}^{+}\right)$if and only if $\omega^{n} \hat{s}(\omega) \in L^{2}(\mathbb{R})$. The assertion (ii) is well known in the Sobolev space theory whose proof is omitted.

Definition 2.2 We say that $s$ has the non-tangential boundary derivative or, in brief, boundary derivative $s^{\prime}(t)$ at $t \in \mathbb{R}$, if a finite limit $s(t)=\lim _{\Gamma^{ \pm}: z \rightarrow t} f(z)$ exists, and

$$
\lim _{\Gamma^{ \pm}: z \rightarrow t} \frac{s(z)-s(t)}{z-t} \triangleq s^{\prime}(t)
$$

The limit allows the infinite values. Replacing $s$ by $s^{(k)}$ in the above definition, we can inductively define the $(k+1)$-order non-tangential boundary derivative $s^{(k+1)}$.

So far the notation $s^{\prime}(t)$ has two meanings. One is the boundary derivative defined by (2.19) and the other is the limit

$$
\lim _{\Gamma^{ \pm}: z \rightarrow t} s^{\prime}(z)
$$

(the fourth type derivative, or analytic derivative). Thanks to the following lemma the notation does not cause ambiguity.

Lemma 2.3 Let $s \in L_{1}^{2}(\mathbb{R})$. Then

(i) Analytic derivative $s^{\prime}$ defined by (2.20) exists.

(ii) Boundary derivative $s^{\prime}$ defined by (2.19) exists as a function in $L^{2}(\mathbb{R})$ that coincides with the analytic derivative.

Proof of Lemma By invoking (i) of Lemma 2.1, assertion (i) is a consequence of the basic result 2. To prove (ii) we use the same method as to prove the counterpart result for the disc case (see [15], page 79 ).

Combining (ii) of Lemma 2.1 and Lemma 2.3, we have

Corollary 2.4 Let $s$ and $s^{(n)}$ belong to $H^{2}\left(\mathbb{C}^{ \pm}\right), n \geq 1$. Then

(i) All the boundary derivatives $s^{\prime}, \ldots, s^{(n)}$ defined through (2.19) exist.

(ii) The boundary derivatives coincide with the corresponding analytic derivatives defined through (2.20), and they all belong to $L^{2}(\mathbb{R})$.

Let $s \in L^{2}(\mathbb{R})$. As a temperate distribution, $s$ has a distributional derivative $\frac{d^{*} s}{d t}$. What is interested is the case when the temperate distribution $\frac{d^{*} s}{d t}$ belongs to $L^{2}(\mathbb{R})$. The latter means that there exists a function $h \in L^{2}(\mathbb{R})$ such that for all $\phi \in \mathcal{S}$, the Schwarz class of rapidly decreasing functions, there holds

$$
<h, \phi>=-<s, \phi^{\prime}>,
$$

and $\frac{d^{*} s}{d t}=h(t)$. We have the following result. 
Lemma 2.5 Let $s \in L^{2}(\mathbb{R})$ and $n$ a positive integer. Then the following conditions are equivalent. (i) $\omega^{n} \hat{s} \in L^{2}(\mathbb{R})$.

(ii) $\left(\frac{d^{*}}{d t}\right)^{n} s$ exists in $L^{2}(\mathbb{R})$.

When they hold, we have $\left(\left(\frac{d^{*}}{d t}\right)^{n} s\right) \wedge(\omega)=(i \omega)^{n} \hat{s}(\omega)$.

This result is well known and proved by standard techniques ([16]). Below we provide a proof by using the Hardy spaces decomposition.

Proof of Lemma We first show (i) implies (ii). Assume $\omega^{n} \hat{s} \in L^{2}(\mathbb{R})$. Then $\omega^{n} \hat{s^{+}}$and $\omega^{n} \hat{s^{-}}$both are in $L^{2}(\mathbb{R})$, where $s^{ \pm} \in H^{2}\left(\mathbb{C}^{ \pm}\right)$. Due to (2.18), we have, for all $y>0$,

$$
\begin{aligned}
\int_{-\infty}^{\infty}\left|s^{+(n)}(t+i y)\right|^{2} d t & =\int_{0}^{\infty} \omega^{2 n} e^{-2 y \omega}\left|\left(s^{+}\right)^{\wedge}(\omega)\right|^{2} d \omega \\
& \leq \int_{0}^{\infty} \omega^{2 n}\left|\left(s^{+}\right)^{\wedge}(\omega)\right|^{2} d \omega \\
& <\infty .
\end{aligned}
$$

This shows that $s^{+(n)} \in H^{2}\left(\mathbb{C}^{+}\right)$and therefore $\left(s^{+(n)}\right)_{y}$ has a $L^{2}(\mathbb{R})$ limit, $h^{+}$, as $y \rightarrow 0+([8]$, p57, Theorem 3.1). On the other hand, for every $y>0$ and $\phi \in \mathcal{S}$,

$$
<\left(s^{+(n)}\right)_{y}, \phi>=<\left(s^{+}\right)_{y},(-1)^{n} \phi^{(n)}>.
$$

Passing to the $L^{2}$-limits of $\left(s^{+(n)}\right)_{y}$ and $\left(s^{+}\right)_{y}$, we obtain

$$
<h^{+}, \phi>=<s^{+},(-1)^{n} \phi^{(n)}>\text {. }
$$

This shows that $\left(\frac{d^{*}}{d t}\right)^{n}\left(s^{+}\right)=h^{+} \in L^{2}(\mathbb{R})$. Similarly we can show that there exists $h^{-} \in L^{2}(\mathbb{R})$ such that $\left(\frac{d^{*}}{d t}\right)^{n}\left(s^{-}\right)=h^{-} \in L^{2}(\mathbb{R})$. Therefore, we have $\left(\frac{d^{*}}{d t}\right)^{n}(s)=h^{+}+h^{-} \triangleq h \in L^{2}(\mathbb{R})$. The proof that (ii) implies (i) is direct. We choose to include it for the completeness. We assume that $\left(\frac{d^{*}}{d t}\right)^{n} s$ exists in the $L^{2}(\mathbb{R})$. There holds

$$
<\left(\frac{d^{*}}{d t}\right)^{n}(s), \phi>=<s,(-1)^{n} \phi^{(n)}>
$$

Using Parseval's identity, we have

$$
<\left(\left(\frac{d^{*}}{d t}\right)^{n} s\right) \hat{\phi}, \hat{\phi}>=<\hat{s},(-i(\cdot))^{n} \hat{\phi}>=<(i(\cdot))^{n} \hat{s}, \hat{\phi}>.
$$

Since $\hat{\phi}$ runs over all $\mathcal{S}$, the boundedness of the left-hand-side shows $(i(\cdot))^{n} \hat{s} \in L^{2}(\mathbb{R})$. This proves that (ii) implies (i), and $\left(\left(\frac{d^{*}}{d t}\right)^{n} s\right) \wedge(\omega)=(i \omega)^{n} \hat{s}(\omega)$.

Lemma 2.6 Let $s \in L^{2}(\mathbb{R})$ and $n$ a positive integer. Then the following assertions hold.

(i) $\omega^{n} \hat{s}(\omega) \in L^{2}(\mathbb{R})$ if and only if $\omega^{n} \hat{s}^{+}(\omega) \in L^{2}(\mathbb{R})$ and $\omega^{n} \hat{s}^{-}(\omega) \in L^{2}(\mathbb{R})$.

(ii) $\left(\frac{d^{*}}{d t}\right)^{n} s \in L^{2}(\mathbb{R})$ if and only if $\left(\frac{d^{*}}{d t}\right)^{n} s^{+} \in L^{2}(\mathbb{R})$ and $\left(\frac{d^{*}}{d t}\right)^{n} s^{-} \in L^{2}(\mathbb{R})$.

(iii) $t^{n} s(t) \in L^{2}(\mathbb{R})$ if and only if $t^{n} s^{+}(t) \in L^{2}(\mathbb{R})$ and $t^{n} s^{-}(t) \in L^{2}(\mathbb{R})$. 
Proof of Lemma (i) is a consequence of $\hat{s^{ \pm}}=\chi_{ \pm} \hat{s}$. (ii) is a consequence of (i) and Lemma 2.5. Now we prove (iii). If $t^{n} s(t) \in L^{2}(\mathbb{R})$, then by Lemma $2.5\left(\frac{d^{*}}{d \omega}\right)^{n} \hat{s}(\omega) \in L^{2}(\mathbb{R})$, and then $\chi_{+}(\omega)\left(\frac{d^{*}}{d \omega}\right)^{n} \hat{s}(\omega)$ and $\chi_{-}(\omega)\left(\frac{d^{*}}{d \omega}\right)^{n} \hat{s}(\omega) \in L^{2}(\mathbb{R})$. We show that we can exchange the order of multiplying by $\chi_{ \pm}$and taking the distributional derivative $\left(\frac{d^{*}}{d \omega}\right)^{n}$. Let $h \in L^{2}(\mathbb{R})$ be such that

$$
<h, \varphi>=<(-1)^{n} \hat{s}, \varphi^{(n)}>, \quad \varphi \in \mathcal{S} .
$$

It is easy to show

$$
<\chi_{ \pm} h, \varphi>=<h, \chi_{ \pm} \varphi>
$$

and

$$
<(-1)^{n} \hat{s},\left(\chi_{ \pm} \varphi\right)^{(n)}>=<(-1)^{n} \hat{s}, \chi_{ \pm} \varphi^{(n)}>=<(-1)^{n} \chi_{ \pm} \hat{s}, \varphi^{(n)}>.
$$

In order to show

$$
<\chi_{ \pm} h, \varphi>=<(-1)^{n} \chi_{ \pm} \hat{s}, \varphi^{(n)}>, \quad \varphi \in \mathcal{S},
$$

that is equivalent to the exchange rule

$$
\chi_{ \pm}\left(\frac{d^{*}}{d \omega}\right)^{n} \hat{s}=\left(\frac{d^{*}}{d \omega}\right)^{n}\left(s^{ \pm} \hat{)}\right.
$$

it suffices to show

$$
<h, \chi_{ \pm} \varphi>=<(-1)^{n} \hat{s},\left(\chi_{ \pm} \varphi\right)^{(n)}>, \quad \varphi \in \mathcal{S} .
$$

Note that $\chi_{ \pm} \varphi \in L_{n}^{2}(\mathbb{R})$. Since the Schwarz class is dense in the Sobolev space $L_{n}^{2}(\mathbb{R})$ (Proposition 1 , p122, [23]), there exists a sequence of functions $\psi_{k}$ in the Schwarz class $\mathcal{S}$ such that in the $L^{2}$ convergence sense

$$
\psi_{k} \rightarrow \chi_{ \pm} \varphi \quad \text { and } \quad \psi_{k}^{(n)} \rightarrow\left(\chi_{ \pm} \varphi\right)^{(n)}
$$

simultaneously. In (2.23) let $\varphi=\psi_{k}$ and take limit $k \rightarrow \infty$, we obtain (2.25). By taking Fourier transform and using the Plancherel Theorem on the right-hand-side of (2.24), we obtain $t^{n} s^{+}(t), t^{n} s^{-}(t) \in$ $L^{2}(\mathbb{R})$. Conversely, if $t^{n} s^{+}(t), t^{n} s^{-}(t) \in L^{2}(\mathbb{R})$, then by adding them up we obtain $t^{n} s(t) \in L^{2}(\mathbb{R})$.

\section{Definition of Phase and Amplitude Derivatives}

Now we proceed to define phase and amplitude derivatives. We first have the following observations. Let $s(z)$ be an analytic function in $\mathbb{C}^{ \pm}$and $s(z)=A_{y}(t) e^{i \varphi_{y}(t)}, z=t+i y, \pm y>0$. Taking the partial derivative with respect to $t$ and dividing $s(t+i y)$ on the both sides, we obtain

$$
\begin{gathered}
\operatorname{Im}\left(\frac{s^{\prime}(z)}{s(z)}\right)=\frac{\partial \varphi_{y}(t)}{\partial t}, \\
A_{y}(t) \operatorname{Re}\left(\frac{s^{\prime}(z)}{s(z)}\right)=\frac{\partial A_{y}(t)}{\partial t} .
\end{gathered}
$$

For a signal in the Sobolev space $L_{1}^{2}(\mathbb{R})$ these relations do not hold on $\mathbb{R}$ in general. It, however, suggests the following formulation. 
Let $s(z)$ be a function analytic in $\mathbb{C}^{ \pm}$. If $t_{0} \in \mathbb{R}$, we denote

$$
D_{p}^{ \pm} s\left(t_{0}\right) \triangleq \lim _{\Gamma: z \rightarrow t_{0}} \operatorname{Im}\left(\frac{s^{\prime}(z)}{s(z)}\right)
$$

and

$$
D_{a}^{ \pm} s\left(t_{0}\right) \triangleq \lim _{\Gamma: z \rightarrow t_{0}} A_{y}^{ \pm}(t) \operatorname{Re}\left(\frac{s^{\prime}(z)}{s(z)}\right)
$$

provided that the limits exist.

Theorem 3.1 If $s, s^{\prime} \in H^{2}\left(\mathbb{C}^{ \pm}\right)$, then both $D_{p}^{ \pm} s$ and $D_{a}^{ \pm} s$ are well defined measurable functions, and

$$
D_{p}^{ \pm} s(t)=\operatorname{Im}\left(\frac{s^{\prime}(t)}{s(t)}\right), \quad D_{a}^{ \pm} s(t)=A^{ \pm}(t) \operatorname{Re}\left(\frac{s^{\prime}(t)}{s(t)}\right), \quad \text { a.e. }
$$

Proof of Theorem We will only prove the result for $s, s^{\prime} \in H^{2}\left(\mathbb{C}^{+}\right)$. If the non-tangential limit of $s(z)$ as $z$ tends to $t$ exists, then the non-tangential limit

$$
A(t)=\lim _{\Gamma: z \rightarrow t} A_{y}(t)=\lim _{\Gamma: z \rightarrow t}\left|s_{y}(t)\right|
$$

also exists. Noticing that $s^{\prime} / s$ is meromorphic, it then suffices to show (i) the non-tangential boundary limits $s(t)$ and $s^{\prime}(t)$ both exist a.e.; and (ii) $s(t)$ is a.e. non-zero. Now (i) follows from the fact that $s(z)$ and $s^{\prime}(z)$ are in the Hardy space $H^{2}\left(\mathbb{C}^{+}\right)$. (ii) follows from a result on $\mathrm{p} 65$, [8], asserting that the non-tangential boundary value of a function in the Hardy $H^{2}\left(\mathbb{C}^{ \pm}\right)$is a.e. non-zero.

Definition 3.2 If $s, s^{\prime} \in H^{2}\left(\mathbb{C}^{ \pm}\right)$, then $s$ has non-tangential boundary limit $s(t)=A^{ \pm}(t) e^{i \varphi^{ \pm}(t)}$. According to Theorem 3.1, $D_{p}^{ \pm} s$ and $D_{a}^{ \pm} s$ both exist as measurable functions. The analytic amplitude derivative (amplitude derivative) and analytic phase derivative (phase derivative) are, respectively, defined to be

$$
A^{ \pm \prime}(t) \triangleq D_{a}^{ \pm} s(t), \quad \varphi^{ \pm \prime}(t) \triangleq D_{p}^{ \pm} s(t)
$$

Remark 3.3 Note that although $\varphi^{ \pm \prime}(t)$ have been defined, phase functions $\varphi^{ \pm}(t)$ are not yet defined. The unimodular functions $e^{i \varphi^{ \pm}(t)}$ are defined by $s^{ \pm}(t) /\left|s^{ \pm}(t)\right|$. This does not mean there exist appropriate parametrizations $\varphi^{ \pm}(t)$ such that $\frac{d \varphi^{ \pm}(t)}{d t}$ are defined in the classical sense. Nevertheless, in the sense specified in Definition 3.2, Theorem 3.1 asserts existence of the phase and the amplitude derivatives of $s^{+}$and $s^{-}$, if $s \in L_{1}^{2}(\mathbb{R})$. On the other hand, it may be seen that if the function $s$ has analytic continuation across an open interval containing $t_{0}$, then the above defined $A^{ \pm^{\prime}}\left(t_{0}\right)$ and $\varphi^{ \pm \prime}\left(t_{0}\right)$ coincide with the respective classical derivatives at $t_{0}$ (also see [18]).

The results proved in this and the last section are summarized as follows.

Proposition 3.4 Let $n$ be a positive integer. Then

(i) If $s, s^{(n)} \in H^{2}\left(\mathbb{C}^{ \pm}\right)$, then $s^{(n)}$, as boundary derivative defined by (2.19) exists, and coincides with the analytic derivative $s^{(n)}$ defined by (2.20) from inside $\mathbb{C}^{ \pm}$. It also coincides with the distributional derivative $\frac{\left(d^{*}\right)^{n} s}{d t^{n}}$. These derivatives are all in $L^{2}(\mathbb{R})$.

(ii) The conditions assumed in (i) are equivalent to the conditions supp $\hat{s} \subset[0, \pm \infty)$, and $\hat{s}(\omega), \omega^{n} \hat{s}(\omega) \in$ $L^{2}(\mathbb{R})$. 
(iii) If the conditions of (i) hold, then the same results hold for $k=1, \ldots, n-1$.

(iv) If $s, s^{\prime} \in H^{2}\left(\mathbb{C}^{ \pm}\right)$, then for the non-tangential boundary value $s(t)=A(t) e^{i \varphi(t)}$, the analytic amplitude and phase derivatives can be defined through non-tangential boundary values of the same quantities from inside of the domain $\mathbb{C}^{ \pm}$, as

$$
A^{\prime}=D_{a} s \quad \text { and } \quad \varphi^{\prime}=D_{p} s .
$$

(v) $\left(\frac{d^{*}}{d t}\right)^{n} s \in L^{2}(\mathbb{R})$ if and only if $\left(\frac{d^{*}}{d t}\right)^{n} s^{+} \in L^{2}(\mathbb{R})$ and $\left(\frac{d^{*}}{d t}\right)^{n} s^{-} \in L^{2}(\mathbb{R})$. In the case, $\left(\frac{d^{*}}{d t}\right)^{n} s=$ $\left(\frac{d^{*}}{d t}\right)^{n} s^{+}+\left(\frac{d^{*}}{d t}\right)^{n} s^{-}$.

(vi) $t^{n} s(t) \in L^{2}(\mathbb{R})$ if and only if $t^{n} s^{+}(t) \in L^{2}(\mathbb{R})$ and $t^{n} s^{-}(t) \in L^{2}(\mathbb{R})$.

(vii) If $s \in L^{2}(\mathbb{R})$, then $\left(\frac{d^{*}}{d t}\right)^{n} s$ exists in $\in L^{2}(\mathbb{R})$ if and only if $\omega^{n} \hat{s}(\omega) \in L^{2}(\mathbb{R})$, and in the case $\left(\left(\frac{d^{*}}{d t}\right)^{n} s\right)^{\wedge}(\omega)=(i \omega)^{n} \hat{s}(\omega)$.

\section{Mean and Variance of Fourier Frequency in Terms of Analytic Phase and Amplitude Derivatives}

Definition 4.1 Let $s$ be a square-integrable signal and $|\hat{s}(\omega)|^{2}$ the density of the Fourier frequency, then we can define the mean of the Fourier frequency by

$$
\langle\omega\rangle \triangleq \int_{-\infty}^{\infty} \omega|\hat{s}(\omega)|^{2} d \omega,
$$

the Fourier bandwidth by

$$
\begin{aligned}
B^{2}=\sigma_{\omega}^{2} & \triangleq \int_{-\infty}^{\infty}(\omega-\langle\omega\rangle)^{2}|\hat{s}(\omega)|^{2} d \omega \\
& =\left\langle\omega^{2}\right\rangle-\langle\omega\rangle^{2}
\end{aligned}
$$

and the mean of any Fourier frequency function $g(\omega)$ by

$$
\langle g(\omega)\rangle \triangleq \int_{-\infty}^{\infty} g(\omega)|\hat{s}(\omega)|^{2} d \omega,
$$

provided that the right-hand sides of (4.1), (4.2) and (4.4) are, respectively, well defined integrals. Below we will also use the notation

$$
\langle g(\omega)\rangle_{ \pm} \triangleq \int_{-\infty}^{\infty} g(\omega)\left|\hat{s}^{ \pm}(\omega)\right|^{2} d \omega
$$

where $\hat{s}^{ \pm}(\omega)$ are defined by (5.7).

With the preparations made in the proceeding section the following theorem is straightforward.

Theorem 4.2 Assume $s, \frac{d^{*}}{d t} s \in L^{2}(R)$. With the decomposition $s=s^{+}+s^{-},\left(s^{ \pm}\right)^{\wedge}=\chi_{ \pm} \hat{s}, s^{ \pm}(t)=$ $A^{ \pm}(t) e^{i \varphi^{ \pm}(t)}$, the mean Fourier frequency defined by (4.1) is identical with

$$
\langle\omega\rangle=\int_{-\infty}^{\infty} \varphi^{+^{\prime}}(t) A^{+2}(t) d t+\int_{-\infty}^{\infty} \varphi^{-\prime}(t) A^{-2}(t) d t,
$$

where $\varphi^{ \pm^{\prime}}(t)$ are defined by (3.2). 
Proof of Theorem Since $s, \frac{d^{*}}{d t} s \in L^{2}(\mathbb{R})$, (vii) of Proposition 3.4 implies that $\hat{s}(\omega), \omega \hat{s}(\omega) \in L^{2}(\mathbb{R})$. Hölder inequality then implies $\omega|\hat{s}(\omega)|^{2} \in L^{1}(\mathbb{R})$, and hence $\langle\omega\rangle$ is well defined. The assertions (v) and (ii) of Proposition 3.4 imply that $s^{ \pm}, s^{ \pm \prime}$ belong to $H^{2}\left(\mathbb{C}^{ \pm}\right)$, respectively. The assertions (i) and (iv) of Proposition 3.4 further imply that the boundary values $s^{ \pm^{\prime}}$ and the phase derivatives $\varphi^{ \pm^{\prime}}$ all exist. There holds the decomposition

$$
\langle\omega\rangle=\langle\omega\rangle^{+}+\langle\omega\rangle^{-},
$$

where

$$
\begin{aligned}
\langle\omega\rangle^{ \pm \triangleq \pm} \int_{0}^{ \pm \infty} \omega|\hat{s}(\omega)|^{2} d \omega & =-\int_{-\infty}^{\infty} i s^{ \pm^{\prime}}(t) \overline{s^{ \pm}(t)} d t \\
& =-i \int_{-\infty}^{\infty} \frac{s^{ \pm^{\prime}}(t)}{s^{ \pm}(t)}\left|s^{ \pm}(t)\right|^{2} d t \\
& =\int_{-\infty}^{\infty} \operatorname{Im}\left\{\frac{s^{ \pm^{\prime}}(t)}{s^{ \pm}(t)}\right\}\left|s^{ \pm}(t)\right|^{2} d t \\
& =\int_{-\infty}^{\infty} \varphi^{ \pm^{\prime}}(t)\left|s^{ \pm}(t)\right|^{2} d t,
\end{aligned}
$$

where the fact that the boundary values $s^{ \pm}$are a.e. non zero $(\mathrm{p} 65,[8])$ justifies the division by $s^{ \pm}(t)$. As consequence,

$$
\langle\omega\rangle=\int_{-\infty}^{\infty} \varphi^{+^{\prime}}(t)\left|s^{+}(t)\right|^{2} d t+\int_{-\infty}^{\infty} \varphi^{-\prime}(t)\left|s^{-}(t)\right|^{2} d t .
$$

Example 4.3 Let $s(t)=\frac{2}{1+t^{2}}$. It has the decomposition $s=s^{+}+s^{-}=\frac{1}{1-i t}+\frac{1}{1+i t}$. Then

$$
\begin{aligned}
A^{ \pm 2}(t)=\left|s^{ \pm}(t)\right|^{2} & =\frac{1}{1+t^{2}} \in L^{2}(\mathbb{R}), \quad s^{ \pm^{\prime}}(t)=\frac{ \pm i}{(1 \mp i t)^{2}} \in L^{2}(\mathbb{R}), \\
\hat{s^{ \pm}}(\omega) & =\sqrt{2 \pi} \chi_{ \pm}(\omega) e^{-|\omega|}, \quad \omega s^{ \pm}(\omega)=\sqrt{2 \pi} \omega \chi_{ \pm}(\omega) e^{-|\omega|} \in L^{2}(\mathbb{R}),
\end{aligned}
$$

and $s^{ \pm}$as analytic functions in $\mathbb{C}^{ \pm}$, respectively, have analytic continuations, and thus have smooth phase derivatives. Precisely,

$$
s^{ \pm}(t)=\frac{1}{1 \mp i t}=\frac{1}{\sqrt{1+t^{2}}} e^{ \pm i \arctan t}
$$

with

$$
\varphi^{ \pm}(t)= \pm \arctan t \quad \text { and } \quad \varphi^{ \pm \prime}(t)=\frac{ \pm 1}{1+t^{2}} .
$$

On the other hand, we also have

$$
\lim _{y \rightarrow 0+} \varphi_{y}^{ \pm^{\prime}}(t)=\lim _{\Gamma: z \rightarrow t} \operatorname{Im}\left(\frac{s^{ \pm^{\prime}}(z)}{s^{ \pm}(z)}\right)=\lim _{\Gamma: z \rightarrow t} \operatorname{Im}\left(\frac{ \pm i}{1 \mp i z}\right)=\frac{ \pm 1}{1+t^{2}},
$$

justifying existence of the phase derivative defined in Definition 3.2. Since $s$ itself is real-valued, $s(t)=\frac{2}{1+t^{2}} e^{i \varphi(t)}$ with $\varphi(t)=2 \pi k$ for any integer $k$. Therefore, $s$ satisfies all the conditions assumed in Theorem 1.1 and Theorem 4.2. By using Theorem 1.1,

$$
\langle\omega\rangle=\int_{-\infty}^{\infty} \varphi^{\prime}(t) A^{2}(t) d t=\int_{-\infty}^{\infty} 0\left(\frac{2}{1+t^{2}}\right)^{2} d t=0 .
$$


This can also be obtained directly from the definition of $\langle\omega\rangle$ and the property $\hat{s}(-\omega)=\overline{\hat{s}(\omega)}$ for real valued signals. By using Theorem 4.2, we have

$$
\begin{aligned}
\langle\omega\rangle & =\langle\omega\rangle^{+}+\langle\omega\rangle^{-} \\
& =\int_{-\infty}^{\infty} \varphi^{+^{\prime}}(t) A^{+2}(t) d t+\int_{-\infty}^{\infty} \varphi^{-\prime}(t) A^{-2}(t) d t \\
& =0 .
\end{aligned}
$$

The results obtained from Theorem 1.1 and Theorem 4.2 coincide.

The following theorem gives a similar formula for $\left\langle\omega^{2}\right\rangle$.

Theorem 4.4 Assume $s, \frac{d^{*}}{d t} s \in L^{2}(\mathbb{R})$. With the decomposition $s=s^{+}+s^{-},\left(s^{ \pm}\right)^{\wedge}=\chi_{ \pm} \hat{s}, s^{ \pm}(t)=$ $A^{ \pm}(t) e^{i \varphi^{ \pm}(t)}$, there follows

$$
\left\langle\omega^{2}\right\rangle=\int_{-\infty}^{\infty}\left[\left(A^{+^{\prime}}(t)\right)^{2}+\left(A^{-^{\prime}}(t)\right)^{2}\right] d t+\int_{-\infty}^{\infty}\left[\left(A^{+}(t) \varphi^{+^{\prime}}(t)\right)^{2}+\left(A^{-}(t) \varphi^{-\prime}(t)\right)^{2}\right] d t .
$$

Proof of Theorem Since $s, \frac{d^{*}}{d t} s \in L^{2}(\mathbb{R})$, (ii) of Proposition 3.4 implies $\omega \hat{s}(\omega) \in L^{2}(\mathbb{R})$, and thus $\omega^{2}|\hat{s}(\omega)|^{2} \in L^{1}(\mathbb{R}) .\left\langle\omega^{2}\right\rangle$ is therefore well defined. The assertion (v) and (ii) of Proposition 3.4 imply that $s^{ \pm}, s^{ \pm^{\prime}}$ belong to $H^{2}\left(\mathbb{C}^{ \pm}\right)$, respectively. The assertion (i) and (iv) of Proposition 3.4 further imply that the boundary values $s^{ \pm^{\prime}}$ and the phase derivatives $\varphi^{ \pm \prime}$ all exist. There then holds the decomposition

$$
\left\langle\omega^{2}\right\rangle=\left\langle\omega^{2}\right\rangle^{+}+\left\langle\omega^{2}\right\rangle^{-}
$$

with

$$
\begin{aligned}
\left\langle\omega^{2}\right\rangle^{ \pm} \triangleq \pm \int_{0}^{ \pm \infty} \omega^{2}|\hat{s}(\omega)|^{2} d \omega & =-\int_{-\infty}^{\infty} i s^{ \pm^{\prime}}(t) \overline{-i s^{ \pm^{\prime}}(t)} d t \\
& =\int_{-\infty}^{\infty}\left|\frac{s^{ \pm^{\prime}}(t)}{s^{ \pm}(t)}\right|^{2}\left|s^{ \pm}(t)\right|^{2} d t \\
& =\int_{-\infty}^{\infty} \operatorname{Re}^{2}\left\{\frac{s^{ \pm^{\prime}}(t)}{s^{ \pm}(t)}\right\}\left|s^{ \pm}(t)\right|^{2} d t+\int_{-\infty}^{\infty} \operatorname{Im}^{2}\left\{\frac{s^{ \pm^{\prime}}(t)}{s^{ \pm}(t)}\right\}\left|s^{+}(t)\right|^{2} d t \\
& =\int_{-\infty}^{\infty}\left[A^{ \pm^{\prime}}(t)\right]^{2} d t+\int_{-\infty}^{\infty}\left[A^{ \pm}(t) \varphi^{ \pm^{\prime}}(t)\right]^{2} d t
\end{aligned}
$$

where, again, the fact that the boundary values $s^{ \pm}$are a.e. non zero (p 65, [8]) justifies the division by $s^{ \pm}(t)$. Therefore,

$$
\left\langle\omega^{2}\right\rangle=\int_{-\infty}^{\infty}\left[\left({A^{+}}^{\prime}(t)\right)^{2}+\left(A^{-^{\prime}}(t)\right)^{2}\right] d t+\int_{-\infty}^{\infty}\left[\left(A^{+}(t) \varphi^{+^{\prime}}(t)\right)^{2}+\left(A^{-}(t) \varphi^{-\prime}(t)\right)^{2}\right] d t .
$$

Corollary 4.5 Assume $s, \frac{d^{*}}{d t} s \in L^{2}(R)$. With the decomposition $s=s^{+}+s^{-},\left(s^{ \pm}\right)^{\wedge}=\chi_{ \pm} \hat{s}, s^{ \pm}(t)=$ $A^{ \pm}(t) e^{i \varphi^{ \pm}(t)}$, the bandwidth

$$
\begin{aligned}
B^{2} & =\left\langle\omega^{2}\right\rangle-\langle\omega\rangle^{2} \\
& =\int_{-\infty}^{\infty}\left[\left({A^{+}}^{\prime}(t)\right)^{2}+\left({A^{-\prime}}^{\prime}(t)\right)^{2}\right] d t+\int_{-\infty}^{\infty}\left[\left(\varphi^{+^{\prime}}(t)-\langle\omega\rangle\right)^{2} A^{+^{2}}(t)+\left(\varphi^{-\prime}(t)-\langle\omega\rangle\right)^{2} A^{-2}(t)\right] d t .
\end{aligned}
$$


Proof of Corollary Based on the expressions of $\langle\omega\rangle,\left\langle\omega^{2}\right\rangle$ obtained in Theorem 4.2 and Theorem 4.4, we have

$$
\begin{aligned}
B^{2}= & \left\langle\omega^{2}\right\rangle-\langle\omega\rangle^{2} \\
= & \left\langle\omega^{2}\right\rangle-2\langle\omega\rangle\left(\langle\omega\rangle^{+}+\langle\omega\rangle^{-}\right)+\langle\omega\rangle^{2} \int_{-\infty}^{\infty}|s(t)|^{2} d t \\
= & \int_{-\infty}^{\infty}\left[\left(A^{+^{\prime}}(t)\right)^{2}+\left(A^{-^{\prime}}(t)\right)^{2}\right] d t+\int_{-\infty}^{\infty}\left[\left(A^{+}(t) \varphi^{+^{\prime}}(t)\right)^{2}+\left(A^{-}(t) \varphi^{-\prime}(t)\right)^{2}\right] d t \\
& \quad-\left[2 \int_{-\infty}^{\infty}\langle\omega\rangle \varphi^{+^{\prime}}(t)\left|s^{+}(t)\right|^{2} d t+2 \int_{-\infty}^{\infty}\langle\omega\rangle \varphi^{-\prime}(t)\left|s^{-}(t)\right|^{2} d t\right] \\
& \quad+\left[\int_{-\infty}^{\infty}\langle\omega\rangle^{2}\left|s^{+}(t)\right|^{2} d t+\int_{-\infty}^{\infty}\langle\omega\rangle^{2}\left|s^{-}(t)\right|^{2} d t\right] \\
& \quad \int_{-\infty}^{\infty}\left[\left(A^{+^{\prime}}(t)\right)^{2}+\left(A^{-^{\prime}}(t)\right)^{2}\right] d t+\int_{-\infty}^{\infty}\left[\left(\varphi^{+^{\prime}}(t)-\langle\omega\rangle\right)^{2} A^{+^{2}}(t)+\left(\varphi^{-\prime}(t)-\langle\omega\rangle\right)^{2} A^{-2}(t)\right] d t .
\end{aligned}
$$

Alternatively, the bandwidth can be obtained directly by the same steps as in the proof of Theorem 4.4

Remark 4.6 If the signal $s(t)$ satisfies the assumptions of Theorem 1.1, then the decomposition $s=s^{+}+s^{-}$can be performed, and the steps in the proofs of Theorem 4.2, Theorem 4.4 and Corollary 4.10 can be followed. The steps to prove the classical results (1.2), (1.9) and

$$
B^{2}=\int_{-\infty}^{\infty}\left[\frac{d A(t)}{d t}\right]^{2} d t+\int_{-\infty}^{\infty}\left\{\left[\frac{d \varphi(t)}{d t}\right]^{2}-\langle\omega\rangle\right\}^{2} A^{2}(t) d t
$$

can also be followed (see [4]). As result, the quantities $\langle\omega\rangle,\left\langle\omega^{2}\right\rangle$ and $B^{2}$ have the alternative representations, and Theorem 4.2, Theorem 4.4 and Corollary 4.10 are generalizations of (1.2), (1.9) and (4.10).

\section{Mean and Variance of Time in Analytic Phase and Amplitude Derivatives of Fourier Transform of the Signal}

We have written the bandwidth and the mean of the Fourier frequency in terms of the phase and the amplitude derivatives of the signal. The same idea can be used to derive the mean of time and the duration in terms of the phase and the amplitude derivatives of the Fourier transform of the signal.

Definition 5.1 Assume $s \in L^{2}(\mathbb{R})$. Define the mean of time by

$$
\langle t\rangle \triangleq \int_{-\infty}^{\infty} t|s(t)|^{2} d t
$$

the duration by

$$
T^{2}=\sigma_{t}^{2} \triangleq \int_{-\infty}^{\infty}(t-\langle t\rangle)^{2}|s(t)|^{2} d t
$$


and the mean of any time function $g(t)$ by

$$
\langle g(t)\rangle \triangleq \int_{-\infty}^{\infty} g(t)|s(t)|^{2} d t
$$

provided that the right-hand-sides of (5.1), (5.2) and (5.3) are, respectively, well defined integrals. Below we will also use the notation

$$
\langle g(t)\rangle_{ \pm} \triangleq \int_{-\infty}^{\infty} g(t)\left|s^{ \pm}(t)\right|^{2} d t
$$

Classical results associated with the amplitude-phase representation of the Fourier spectrum $\hat{s}$ include (see [4])

$$
\langle t\rangle=-\int_{-\infty}^{\infty} \frac{d \psi(\omega)}{d \omega}|\hat{s}(\omega)|^{2} d \omega
$$

and

$$
T^{2}=\sigma_{t}^{2}=\int_{-\infty}^{\infty}\left(\frac{d B(\omega)}{d \omega}\right)^{2} d \omega+\int_{-\infty}^{\infty}\left(\frac{d \psi(\omega)}{d \omega}+\langle t\rangle\right)^{2} B^{2}(\omega) d \omega
$$

where $\hat{s}(\omega)=B(\omega) e^{i \psi(\omega)}$. Under the classical setting, these results are proved based on the pointwise (strong) differentiability being counterpart to those assumed in Theorem1.1.

Assume $s(t) \in L^{2}(R)$ and $t s(t) \in L^{2}(R)$. Then $\hat{s}(\omega) \in L^{2}(R)$. We write $\hat{s}(\omega)$ as $\hat{s}(\omega)=\hat{s}^{+}(\omega)+$ $\hat{s}^{-}(\omega)$, where

$$
\hat{s}^{+}(\omega)=\left[\chi_{-} s\right]^{\wedge}(\omega), \quad \hat{s}^{-}(\omega)=\left[\chi_{+} s\right]^{\wedge}(\omega) .
$$

Similarly to the case of amplitude-phase representation of the signal $s$, we now have $\hat{s}^{ \pm}(\omega)=B^{ \pm}(\omega) e^{i \psi^{ \pm}(\omega)}$ $\in L^{2}(\mathbb{R})$, where $B^{ \pm^{\prime}}(\omega)$ and $\psi^{ \pm^{\prime}}(\omega)$ are defined by the same way as in Theorem 3.1 and Definition 3.2. We have the following two results.

Theorem 5.2 Assume $s(t) \in L^{2}(R)$ and $t s(t) \in L^{2}(R)$. With the decomposition $\hat{s}(\omega)=\hat{s}^{+}(\omega)+$ $\hat{s}^{-}(\omega), \hat{s}^{ \pm}(\omega)=\left[\chi_{\mp} s\right]^{\wedge}(\omega), \hat{s}^{ \pm}(\omega)=B^{ \pm}(\omega) e^{i \psi^{ \pm}(\omega)}$, the mean time defined by (5.1) is identical with

$$
\langle t\rangle=-\int_{-\infty}^{\infty} \psi^{-\prime}(\omega) B^{-2}(\omega) d \omega-\int_{-\infty}^{\infty} \psi^{+^{\prime}}(\omega) B^{+2}(\omega) d \omega .
$$

Theorem 5.3 Assume $s(t) \in L^{2}(R)$ and $t s(t) \in L^{2}(R)$. With the decomposition $\hat{s}(\omega)=\hat{s}^{+}(\omega)+$ $\hat{s}^{-}(\omega), \hat{s}^{ \pm}(\omega)=\left[\chi_{\mp} s(\cdot)\right]^{\wedge}(\omega), \hat{s}^{ \pm}(\omega)=B^{ \pm}(\omega) e^{i \psi^{ \pm}(\omega)}$, the duration is defined by (5.2) is identical with

$\sigma_{t}^{2}=\int_{-\infty}^{\infty}\left[\left(B^{-\prime}(\omega)\right)^{2}+\left(B^{+^{\prime}}(\omega)\right)^{2}\right] d \omega+\int_{-\infty}^{\infty}\left[\left(\psi^{-\prime}(\omega)+\langle t\rangle\right)^{2} B^{-2}(\omega)+\left({\psi^{+}}^{\prime}(\omega)+\langle t\rangle\right)^{2} B^{+^{2}}(\omega)\right] d \omega$.

The proofs of Theorem 5.2 and Theorem 5.3 are omitted as they are similar to those of Theorem 4.2 and Theorem 4.4. 


\section{Covariance Under Hardy-Sobolev Spaces Decomposition}

It is well known that correlation between frequency and time is measured by the covariance given in

Definition 6.1 [4] Let $s(t)=A(t) e^{i \varphi(t)} \in L^{2}(\mathbb{R})$, where $A(t)=|s(t)|$. Assume that the classical derivatives $\frac{d A(t)}{d t}, \frac{d \varphi(t)}{d t}$ and $\frac{d s(t)}{d t}$ all exist as Lebesgue measurable functions, and $\frac{d s}{d t}$ is in $L^{2}(\mathbb{R})$. The covariance is defined by

$$
\operatorname{Cov}_{\mathrm{t} \omega}=\left\langle t \frac{d}{d t} \varphi(t)\right\rangle-\langle t\rangle\langle\omega\rangle
$$

This definition is based on existence of the classical derivatives $\frac{d A}{d t}, \frac{d s}{d t}$ and $\frac{d \varphi}{d t}$. The following is an extension of the above definition to general cases.

Definition 6.2 Assume $s, \frac{d^{*}}{d t} s \in L^{2}(R)$. With the decomposition $s=s^{+}+s^{-},\left(s^{ \pm}\right)^{\wedge}(\omega)=\chi_{ \pm} \hat{s}(\omega), s^{ \pm}(t)=$ $A^{ \pm}(t) e^{i \varphi^{ \pm}(t)}$, the covariance is defined by

$$
\operatorname{Cov}_{\mathrm{t} \omega}=\left\langle t \varphi^{+^{\prime}}(t)\right\rangle_{+}+\left\langle t \varphi^{-1}(t)\right\rangle_{-}-\langle t\rangle\langle\omega\rangle .
$$

Proposition 6.3 Assume that signal s satisfies the conditions in Definition 6.1. Then it also satisfies the conditions in Definition 6.2, and the right-hand-side of equation (6.2) reduces to the right-hand-side of equation (6.1).

Proof of Proposition Since strong differentiability implies weak differentiability (see Remark 8.3), the signal $s(t)$ also satisfies the conditions in Definition 6.2. The phase derivatives $\varphi^{ \pm \prime}(t)=\operatorname{Im}\left\{\frac{s^{ \pm^{\prime}}(t)}{s^{ \pm}(t)}\right\}$ are well defined, and

$$
\begin{aligned}
\left\langle t \varphi^{ \pm^{\prime}}(t)\right\rangle_{ \pm} & =\int_{-\infty}^{\infty} t \varphi^{ \pm^{\prime}}(t)\left|s^{ \pm}(t)\right|^{2} d t \\
& =\operatorname{Im} \int_{-\infty}^{\infty} s^{ \pm^{\prime}}(t) \overline{t s^{ \pm}(t)} d t \\
& =\operatorname{Im} \int_{-\infty}^{\infty} i \omega\left(s^{ \pm}\right)^{\wedge}(\omega) \overline{i\left(s^{ \pm}\right)^{\wedge}(\omega)} d \omega \\
& =\operatorname{Im} \int_{-\infty}^{\infty} \omega\left(s^{ \pm}\right)^{\wedge}(\omega) \overline{\left(s^{ \pm}\right)^{\wedge}(\omega)} d \omega .
\end{aligned}
$$

Therefore,

$$
\begin{aligned}
\left\langle t \varphi^{+^{\prime}}(t)\right\rangle_{+}+\left\langle t \varphi^{-\prime}(t)\right\rangle_{-} & =\operatorname{Im} \int_{-\infty}^{\infty} \omega\left(s^{+}\right)^{\wedge}(\omega) \overline{\left(s^{+}\right)^{\wedge}(\omega)} d \omega+\operatorname{Im} \int_{-\infty}^{\infty} \omega\left(s^{-}\right)^{\wedge}(\omega) \overline{\left(s^{-}\right)^{\wedge}(\omega)} d \omega \\
& =\operatorname{Im} \int_{0}^{\infty} \omega \hat{s}(\omega) \overline{\hat{s}^{\prime}(\omega)} d \omega+\operatorname{Im} \int_{-\infty}^{0} \omega \hat{s}(\omega) \overline{\hat{s}^{\prime}(\omega)} d \omega \\
& =\operatorname{Im} \int_{-\infty}^{\infty} \omega \hat{s}(\omega) \overline{\hat{s}^{\prime}(\omega)} d \omega .
\end{aligned}
$$


Since $s$ satisfies the assumptions in Definition 6.1, $\varphi^{\prime}(t)$ is well defined and identical with $\operatorname{Im}\left\{\frac{s^{\prime}(t)}{s(t)}\right\}$. Therefore,

$$
\begin{aligned}
\left\langle t \varphi^{\prime}(t)\right\rangle & =\int_{-\infty}^{\infty} t \varphi^{\prime}(t)|s(t)|^{2} d t \\
& =\operatorname{Im} \int_{-\infty}^{\infty} s^{\prime}(t) \overline{t s(t)} d t \\
& =\operatorname{Im} \int_{-\infty}^{\infty} i \omega \hat{s}(\omega) \overline{i \hat{s}(\omega)^{\prime}(\omega)} d \omega \\
& =\operatorname{Im} \int_{-\infty}^{\infty} \omega \hat{s}(\omega) \overline{\hat{s}^{\prime}(\omega)} d \omega
\end{aligned}
$$

and

$$
\left\langle t \varphi^{+^{\prime}}(t)\right\rangle_{+}+\left\langle t \varphi^{-\prime}(t)\right\rangle_{-}=\left\langle t \varphi^{\prime}(t)\right\rangle .
$$

Thus the right-hand-side of the equation (6.2) is identical with the right-hand-side of the equation (6.1).

The covariance defined in Definition 6.2 is with respect to the time domain, where the Fourier frequency $\omega$ is replaced by the analytic phase derivative $\varphi^{ \pm^{\prime}}(t)$. One can alternatively define the covariance with respect to the frequency domain, where the time $t$ should be replaced by the group delay $-\psi^{ \pm}(\omega)$. The following question arises: If we define the covariance by

$$
\operatorname{Cov}_{t \omega}=-\left\langle\omega \psi^{+^{\prime}}(\omega)\right\rangle_{+}-\left\langle\omega \psi^{-\prime}(\omega)\right\rangle_{-}-\langle t\rangle\langle\omega\rangle,
$$

whether the two definitions are equivalent? The following theorem gives the positive answer.

Theorem 6.4 Assume $s(t), \frac{d^{*}}{d t} s, t s(t) \in L^{2}(R)$. With the decomposition $s=s^{+}+s^{-},\left(s^{ \pm}\right)^{\wedge}=$ $\chi_{ \pm} \hat{s}, s^{ \pm}(t)=A^{ \pm}(t) e^{i \varphi^{ \pm}(t)}, \hat{s}(\omega)=\hat{s}^{+}(\omega)+\hat{s}^{-}(\omega), \hat{s}^{ \pm}(\omega)=\left[\chi_{\mp} s\right]^{\wedge}(\omega)$ and $\hat{s}^{ \pm}(\omega)=B^{ \pm}(\omega) e^{i \psi^{ \pm}(\omega)}$, there holds

$$
\left\langle t \varphi^{+^{\prime}}(t)\right\rangle_{+}+\left\langle t \varphi^{-^{\prime}}(t)\right\rangle_{-}=-\left\langle\omega \psi^{+^{\prime}}(\omega)\right\rangle_{+}-\left\langle\omega \psi^{-\prime}(\omega)\right\rangle_{-} .
$$

Proof of Theorem 6.4 Since $s(t)$ and $\frac{d^{*}}{d t} s, t s(t) \in L^{2}(R)$, the phase derivatives of $s^{ \pm}(t)$ and $\hat{s}^{ \pm}(\omega)$, viz. $\varphi^{ \pm^{\prime}}(t)$ and $\psi^{ \pm^{\prime}}(\omega)$, all exist, and $\varphi^{ \pm^{\prime}}(t)=\operatorname{Im}\left\{\frac{s^{ \pm^{\prime}}(t)}{s^{ \pm}(t)}\right\}, \psi^{ \pm^{\prime}}(\omega)=\operatorname{Im}\left\{\frac{\hat{s}^{ \pm^{\prime}}(\omega)}{\hat{s}^{ \pm}(\omega)}\right\}$. 
We have

$$
\begin{aligned}
\int_{-\infty}^{\infty} t \varphi^{ \pm^{\prime}}(t)\left|s^{ \pm}(t)\right|^{2} d t= & \operatorname{Im} \int_{-\infty}^{\infty} s^{ \pm^{\prime}}(t) \overline{t s^{ \pm}(t)} d t \\
= & \operatorname{Im} \int_{-\infty}^{\infty}\left[\chi_{+}(t) s^{ \pm}(t)+\chi_{-}(t) s^{ \pm}(t)\right]^{\prime} \overline{t\left[\chi_{+}(t) s^{ \pm}(t)+\chi_{-}(t) s^{ \pm}(t)\right]} d t \\
= & \operatorname{Im} \int_{-\infty}^{\infty} \chi_{+}(t) s^{ \pm^{\prime}}(t) \overline{t\left[\chi_{+}(t) s^{ \pm}(t)\right]} d t+\operatorname{Im} \int_{-\infty}^{\infty} \chi_{+}(t) s^{ \pm^{\prime}}(t) \overline{t\left[\chi_{-}(t) s^{ \pm}(t)\right]} d t \\
& +\operatorname{Im} \int_{-\infty}^{\infty} \chi_{-}(t) s^{ \pm^{\prime}}(t) \overline{t\left[\chi_{+}(t) s^{ \pm}(t)\right]} d t+\operatorname{Im} \int_{-\infty}^{\infty} \chi_{-}(t) s^{ \pm^{\prime}}(t) \overline{t\left[\chi_{-}(t) s^{ \pm}(t)\right]} d t \\
= & \operatorname{Im} \int_{-\infty}^{\infty} \chi_{+}(t) s^{ \pm \prime}(t) \overline{t\left[\chi_{+}(t) s^{ \pm}(t)\right]} d t+\operatorname{Im} \int_{-\infty}^{\infty} \chi_{-}(t) s^{ \pm \prime}(t) \overline{t\left[\chi_{-}(t) s^{ \pm}(t)\right]} d t \\
= & \operatorname{Im} \int_{-\infty}^{\infty} i \omega\left(\chi_{+} s^{ \pm}\right)^{\wedge}(\omega) \overline{i\left(\chi_{+} s^{ \pm}\right)^{\wedge^{\prime}}(\omega)} d \omega \\
& +\operatorname{Im} \int_{-\infty}^{\infty} i \omega\left(\chi_{-} s^{ \pm}\right)^{\wedge}(\omega) \overline{i\left(\chi_{-} s^{ \pm}\right)^{\wedge^{\prime}}(\omega)} d \omega \\
= & \operatorname{Im} \int_{-\infty}^{\infty} \omega\left(\chi_{+} s^{ \pm}\right)^{\wedge}(\omega) \overline{\left(\chi_{+} s^{ \pm}\right)^{\wedge^{\prime}}(\omega)} d \omega \\
& +\operatorname{Im} \int_{-\infty}^{\infty} \omega\left(\chi_{-} s^{ \pm}\right)^{\wedge}(\omega) \overline{\left(\chi_{-} s^{ \pm}\right)^{\wedge^{\prime}}(\omega)} d \omega
\end{aligned}
$$

where the cross terms vanish because of the relation $\chi_{+} \chi_{-}=0$. Denote

$$
\int_{-\infty}^{\infty} \omega\left(\chi_{+} s^{ \pm}\right)^{\wedge}(\omega) \overline{\left(\chi_{+} s^{ \pm}\right)^{\wedge^{\prime}}(\omega)} d \omega+\int_{-\infty}^{\infty} \omega\left(\chi_{-} s^{ \pm}\right)^{\wedge}(\omega) \overline{\left(\chi_{-} s^{ \pm}\right)^{\wedge^{\prime}}(\omega)} d \omega \triangleq f^{ \pm} .
$$

On the other hand,

$$
\begin{aligned}
\int_{-\infty}^{\infty} \omega \psi^{ \pm \prime}(\omega)\left|\hat{s}^{ \pm}(\omega)\right|^{2} d \omega= & \operatorname{Im} \int_{-\infty}^{\infty} \omega\left(\hat{s}^{ \pm}\right)^{\prime}(\omega) \overline{\hat{s}^{ \pm}(\omega)} d \omega \\
= & \operatorname{Im} \int_{-\infty}^{\infty} \omega\left[\left(\chi_{\mp} s^{+}\right)^{\wedge}(\omega)+\left(\chi_{\mp} s^{-}\right)^{\wedge}(\omega)\right]^{\prime} \\
& \frac{\left(\chi_{\mp} s^{+}\right)^{\wedge}(\omega)+\left(\chi_{\mp} s^{-}\right)^{\wedge}(\omega)}{d} d \omega \\
= & \operatorname{Im} \int_{-\infty}^{\infty} \omega\left(\chi_{\mp} s^{+}\right)^{\wedge^{\prime}}(\omega) \overline{\left(\chi_{\mp} s^{+}\right)^{\wedge}(\omega)} d \omega \\
& +\operatorname{Im} \int_{-\infty}^{\infty} \omega\left(\chi_{\mp} s^{+}\right)^{\wedge^{\prime}}(\omega) \overline{\left(\chi_{\mp} s^{-}\right)^{\wedge}(\omega)} d \omega \\
& +\operatorname{Im} \int_{-\infty}^{\infty} \omega\left(\chi_{\mp} s^{-}\right)^{\wedge^{\prime}}(\omega) \overline{\left(\chi_{\mp} s^{+}\right)^{\wedge}(\omega)} d \omega \\
& +\operatorname{Im} \int_{-\infty}^{\infty} \omega\left(\chi_{\mp} s^{-}\right)^{\wedge^{\prime}}(\omega) \overline{\left(\chi_{\mp} s^{-}\right)^{\wedge}(\omega)} d \omega .
\end{aligned}
$$

By adding

$$
\int_{-\infty}^{\infty} \omega \psi^{+^{\prime}}(\omega)\left|\hat{s}^{+}(\omega)\right|^{2} d \omega \text { and } \int_{-\infty}^{\infty} \omega \psi^{-\prime}(\omega)\left|\hat{s}^{-}(\omega)\right|^{2} d \omega
$$


the cross terms are canceled out. In fact,

$$
\begin{aligned}
& \int_{-\infty}^{\infty} \omega\left(\chi_{-} s^{+}\right)^{\wedge^{\prime}}(\omega) \overline{\left(\chi_{-} s^{-}\right)^{\wedge}(\omega)} d \omega+\int_{-\infty}^{\infty} \omega\left(\chi_{+} s^{+}\right)^{\wedge^{\prime}}(\omega) \overline{\left(\chi_{+} s^{-}\right)^{\wedge}(\omega)} d \omega \\
= & \left.\int_{-\infty}^{\infty}\left[\omega\left(\chi_{-} s^{+}\right)^{\wedge^{\prime}}(\omega)+\omega\left(\chi_{+} s^{+}\right)^{\wedge^{\prime}}(\omega)\right] \overline{\left(\chi_{-} s^{-}\right)^{\wedge}(\omega)}+\overline{\left(\chi_{+} s^{-}\right)^{\wedge}(\omega)}\right] d \omega \\
& -\int_{-\infty}^{\infty} \omega\left(\chi_{-} s^{+}\right)^{\wedge^{\prime}}(\omega) \overline{\left(\chi_{+} s^{-}\right)^{\wedge}(\omega)} d \omega-\int_{-\infty}^{\infty} \omega\left(\chi_{+} s^{+}\right)^{\wedge^{\prime}}(\omega) \overline{\left(\chi_{-} s^{-}\right)^{\wedge}(\omega)} d \omega \\
= & \int_{-\infty}^{\infty} \omega\left(s^{+}\right)^{\wedge^{\prime}}(\omega) \overline{\left(s^{-}\right)^{\wedge}(\omega)} d \omega \\
& -\int_{-\infty}^{\infty}-i t \chi_{-}(t) s^{+}(t) \overline{-i \chi_{+}(t) s^{-^{\prime}}(t)} d t-\int_{-\infty}^{\infty}-i t \chi_{+}(t) s^{+}(t) \overline{-i \chi_{-}(t) s^{-\prime}(t)} d t \\
= & 0 .
\end{aligned}
$$

Similarly,

$$
\int_{-\infty}^{\infty} \omega\left(\chi_{-} s^{-}\right)^{\wedge^{\prime}}(\omega) \overline{\left(\chi_{-} s^{+}\right)^{\wedge}(\omega)} d \omega+\int_{-\infty}^{\infty} \omega\left(\chi_{+} s^{-}\right)^{\wedge^{\prime}}(\omega) \overline{\left(\chi_{+} s^{+}\right)^{\wedge}(\omega)} d \omega=0
$$

Therefore, with the notation

$$
\int_{-\infty}^{\infty} \omega\left(\chi_{\mp} s^{+}\right)^{\wedge^{\prime}}(\omega) \overline{\left(\chi_{\mp} s^{+}\right)^{\wedge}(\omega)} d \omega+\int_{-\infty}^{\infty} \omega\left(\chi_{\mp} s^{-}\right)^{\wedge^{\prime}}(\omega) \overline{\left(\chi_{\mp} s^{-}\right)^{\wedge}(\omega)} d \omega \triangleq g^{ \pm},
$$

we have

$$
\int_{-\infty}^{\infty} \omega \psi^{+^{\prime}}(\omega)\left|\hat{s}^{+}(\omega)\right|^{2} d \omega+\int_{-\infty}^{\infty} \omega \psi^{-\prime}(\omega)\left|\hat{s}^{-}(\omega)\right|^{2} d \omega=\operatorname{Im}\left(g^{+}+g^{-}\right) .
$$

It then follows

$$
f^{+}+f^{-}=\overline{g^{+}+g^{-}} .
$$

Therefore,

$$
\operatorname{Im}\left(f^{+}+f^{-}\right)=-\operatorname{Im}\left(g^{+}+g^{-}\right)
$$

As consequence,

$\int_{-\infty}^{\infty} t \varphi^{+^{\prime}}(t)\left|s^{+}(t)\right|^{2} d t+\int_{-\infty}^{\infty} t \varphi^{-\prime}(t)\left|s^{-}(t)\right|^{2} d t=-\int_{-\infty}^{\infty} \omega \psi^{+^{\prime}}(\omega)\left|\hat{s}^{+}(\omega)\right|^{2} d \omega-\int_{-\infty}^{\infty} \omega \psi^{-{ }^{\prime}}(\omega)\left|\hat{s}^{-}(\omega)\right|^{2} d \omega$,

that is,

$$
\left\langle t \varphi^{+^{\prime}}(t)\right\rangle_{+}+\left\langle t \varphi^{-^{\prime}}(t)\right\rangle_{-}=-\left\langle\omega \psi^{+^{\prime}}(\omega)\right\rangle_{+}-\left\langle\omega \psi^{-^{\prime}}(\omega)\right\rangle_{-} .
$$




\section{Uncertainty Principle Under Hardy-Sobolev Spaces Decomposi- tion}

Next we present a proof of the uncertainty principle by using the Hardy-Sobolev spaces decomposition.

Theorem 7.1 Assume $s(t), \frac{d^{*}}{d t} s, t s(t) \in L^{2}(R)$. Then there holds

$$
\sigma_{t} \sigma_{\omega} \geq \frac{1}{2} \sqrt{1+4 \operatorname{Cov}_{\mathrm{t} \omega}^{2}} .
$$

Proof of Theorem 7.1 For the signal $s(t)$, the new signal defined by

$$
s_{\text {new }}(t)=e^{-i\langle\omega\rangle(t+\langle t\rangle)} s(t+\langle t\rangle)
$$

has the same shape in both time and frequency as $s(t)$ does, except that it has been translated in time and frequency such that the means become zero. Conversely, if we have a signal $s_{\text {new }}$ that has zero mean time and zero mean frequency and we want a signal $s(t)$ of the same shape but with the particular mean time $\langle t\rangle$ and the mean frequency $\langle\omega\rangle$, then

$$
s(t)=e^{i\langle\omega\rangle t} s_{\text {new }}(t-\langle t\rangle) .
$$

So we may assume $\langle t\rangle=0,\langle\omega\rangle=0$, and the bandwidth is

$$
\sigma_{\omega}^{2}=\int_{-\infty}^{\infty} \omega^{2}|\hat{s}(\omega)|^{2} d \omega=\int_{-\infty}^{\infty}\left|\frac{d^{*}}{d t} s(t)\right|^{2} d t
$$

and the duration is

$$
\sigma_{t}^{2}=\int_{-\infty}^{\infty} t^{2}|s(t)|^{2} d t
$$

By using Hölder's inequality and (v) and (vi) of Proposition 3.4, we have

$$
\begin{aligned}
& \sigma_{t}^{2} \sigma_{\omega}^{2}=\int_{-\infty}^{\infty} t^{2}|s(t)|^{2} d t \times \int_{-\infty}^{\infty}\left|\frac{d^{*}}{d t} s(t)\right|^{2} d t \\
& \geq\left|\int_{-\infty}^{\infty} \overline{t s(t)} \frac{d^{*}}{d t} s(t) d t\right|^{2} \\
& =\left|\int_{-\infty}^{\infty} \overline{t\left[s^{+}(t)+s^{-}(t)\right]}\left[s^{+^{\prime}}(t)+s^{-\prime}(t)\right] d t\right|^{2} \\
& =\left|\int_{-\infty}^{\infty} \overline{t s^{+}(t)} s^{+^{\prime}}(t) d t+\int_{-\infty}^{\infty} \overline{t s^{-}(t)} s^{-{ }^{\prime}}(t) d t\right|^{2} .
\end{aligned}
$$

With $s^{+}(t+i y)=s_{y}^{+}(t)=A_{y}^{+}(t) e^{i \varphi_{y}^{+}(t)}, y>0$, the product rule of classical derivative gives

$$
\begin{aligned}
\overline{t s_{y}^{+}(t)} s_{y}^{+^{\prime}}(t) & =t A_{y}^{+}(t) A_{y}^{+^{\prime}}(t)+i t A_{y}^{+2}(t) \varphi_{y}^{+^{\prime}}(t) \\
& =\frac{1}{2}\left(t A_{y}^{+2}(t)\right)^{\prime}-\frac{1}{2} A_{y}^{+^{2}}(t)+i t A_{y}^{+2}(t) \varphi_{y}^{+^{\prime}}(t) .
\end{aligned}
$$

Below the main effort will be to justify the integrability of (7.1) and passing to limit $y \rightarrow 0$. Now we show that $t s_{y}^{+}$is dominated by a $L^{2}(\mathbb{R})$ function independent of $y$. It suffices to show that $z s^{+}(z)=$ 
$(t+i y) s_{y}^{+}(t)$ is a function in $H^{2}\left(\mathbb{C}^{+}\right)$. To prove this we start with showing that the Fourier transform of $t s^{+}(t)$ is supported in $[0, \infty)$. A basic result of Fourier transform gives

$$
\left[(\cdot) s^{+}(\cdot)\right]^{\wedge}(\omega)=i \frac{d^{*}}{d \omega}\left(\chi_{+} s\right)^{\wedge}(\omega)=i \chi_{+}(\omega) \frac{d^{*}}{d \omega} \hat{s}(\omega)
$$

where the validity of exchange of multiplication by $\chi_{+}$and operation of taking distributional derivative is proved in the proof of Lemma 2.6(iii). Thus the Fourier transform of $t s^{+}(t)$ is supported in $[0, \infty)$, and hence $t s^{+}(t)$ is the boundary value of a function $g(z)$ in $H^{2}\left(\mathbb{C}^{+}\right)$. We are to show $g(z)=z s^{+}(z)$. The Hardy function $g(z)$ is given by

$$
\begin{aligned}
g(z) & =\frac{1}{2 \pi i} \int_{-\infty}^{\infty} \frac{x s^{+}(x)}{x-(t+i y)} d x \\
& =\frac{1}{2 \pi i} \int_{-\infty}^{\infty} \sqrt{2 \pi} i e^{i \omega t} e^{-\omega y} \chi_{+}(\omega)\left[(\cdot) s^{+}(\cdot)\right]^{\wedge}(\omega) d \omega \\
& =\frac{i}{\sqrt{2 \pi}} \int_{-\infty}^{\infty} e^{i \omega t} e^{-\omega y} \chi_{+}(\omega) \frac{d^{*}}{d \omega}\left[s^{+}(\cdot)\right]^{\wedge}(\omega) d \omega .
\end{aligned}
$$

Since multiplication by $\chi_{+}$can commute with $\frac{d^{*}}{d \omega}$, and since $e^{i \omega t} e^{-\omega y}$, as a function of $\omega$, is in the Schwarz class, we have

$$
\begin{aligned}
g(z) & =\frac{i}{\sqrt{2 \pi}} \int_{-\infty}^{\infty} e^{i \omega t} e^{-\omega y} \frac{d^{*}}{d \omega}\left[s^{+}(\cdot)\right]^{\wedge}(\omega) d \omega \\
& =-\frac{i}{\sqrt{2 \pi}} \int_{-\infty}^{\infty}\left[e^{i \omega t} e^{-\omega y}\right]_{\omega}^{\prime}\left[s^{+}(\cdot)\right]^{\wedge}(\omega) d \omega \\
& =\frac{z}{\sqrt{2 \pi}} \int_{-\infty}^{\infty} e^{i \omega t} e^{-\omega y}\left[s^{+}(\cdot)\right]^{\wedge}(\omega) d \omega \\
& =z s^{+}(z) .
\end{aligned}
$$

Therefore, $z s^{+}(z)$, and so $t s_{y}^{+}(t)$ as well, are dominated by a non-tangential maximal function in $L^{2}(\mathbb{R})$ independent of $y$. The function $s^{+^{\prime}}(z)=s_{y}^{+^{\prime}}(t)$ belongs to $H^{2}\left(\mathbb{C}^{+}\right)$and is also dominated by the corresponding non-tangential maximal functions in $L^{2}(\mathbb{R})$. Therefore, the product $\overline{t s_{y}^{+}(t)} s_{y}^{+\prime}(t)$ on the left-end of (7.1) is dominated by a function in $L^{1}(\mathbb{R})$ that is independent of $y$. It is easy to show that the terms

$$
A_{y}^{+2}(t) \text { and } t A_{y}^{+2}(t) \varphi_{y}^{+\prime}(t)
$$

are also dominated by $L^{1}(\mathbb{R})$ functions independent of $y$. In fact,

$$
A_{y}^{+2}(t)=\left|s_{y}^{+}\right|^{2} \quad \text { and } \quad t A_{y}^{+2}(t) \varphi_{y}^{+\prime}(t)=t\left|s_{y}^{+}(t)\right|^{2} \operatorname{Im}\left(\frac{\left(s_{y}^{+}\right)^{\prime}(t)}{s_{y}^{+}(t)}\right) .
$$

Therefore, all the terms on the left-end and the right-end of the equation chain (7.1) are dominated by $L^{1}(\mathbb{R})$ functions independent of $y$. Now the integrability of $t A_{y}^{+2}(t)$ implies

$$
\int_{-N}^{M}\left(t A_{y}^{+2}(t)\right)^{\prime} d t=\left(t A_{y}^{+2}(t)\right)_{-N}^{M} \rightarrow 0
$$


for suitably choosing $M, N \rightarrow \infty$. From (7.1) we have

$$
\int_{-\infty}^{\infty} \overline{t s_{y}^{+}(t)} s_{y}^{+^{\prime}}(t) d t=-\frac{1}{2} \int_{-\infty}^{\infty} A_{y}^{+2}(t) d t+i \int_{-\infty}^{\infty} t A_{y}^{+2}(t) \varphi_{y}^{+\prime}(t) d t .
$$

By using Lebesgue's dominated convergence theorem, we have

$$
\int_{-\infty}^{\infty} \overline{t s^{+}(t)} s^{+^{\prime}}(t) d t=-\frac{1}{2} \int_{-\infty}^{\infty} A^{+^{2}}(t) d t+i\left\langle t \varphi^{+^{\prime}}(t)\right\rangle_{+} .
$$

Therefore,

$$
\begin{aligned}
\int_{-\infty}^{\infty} \overline{t s^{+}(t)} s^{+^{\prime}}(t) d t+\int_{-\infty}^{\infty} \overline{t s^{-}(t)} s^{-\prime}(t) d t= & -\frac{1}{2} \int_{-\infty}^{\infty} A^{+^{2}}(t) d t+i\left\langle t \varphi^{+^{\prime}}(t)\right\rangle_{+} \\
& -\frac{1}{2} \int_{-\infty}^{\infty} A^{-2}(t) d t+i\left\langle t \varphi^{-\prime}(t)\right\rangle_{-} \\
= & -\frac{1}{2}+i\left[\left\langle t \varphi^{+^{\prime}}(t)\right\rangle_{+}+\left\langle t \varphi^{-\prime}(t)\right\rangle_{-}\right] \\
= & -\frac{1}{2}+i \operatorname{Cov}_{\mathrm{t} \omega} .
\end{aligned}
$$

Thus,

$$
\sigma_{t}^{2} \sigma_{\omega}^{2} \geq \frac{1}{4}+\operatorname{Cov}_{t \omega}^{2}
$$

that is

$$
\sigma_{t} \sigma_{\omega} \geq \frac{1}{2} \sqrt{1+4 \operatorname{Cov}_{\mathrm{t} \omega}^{2}}
$$

\section{Conclusions and Remarks}

In this paper we show that the analytic phase and amplitude derivatives may be defined for signals in the Hardy-Sobolev spaces $H^{ \pm} L_{1}^{2}(\mathbb{R})$. As application, formulas (1.2), (1.9) and (1.10) can be extended in appropriate forms to signals in the Sobolev space $L_{1}^{2}(\mathbb{R})$. The classical uncertainty principle may be proved by means of Hardy-Sobolev spaces decomposition.

Remark 8.1 There has been unfaded interest in the concept analytic signal since Gabor first introduced it ([7]). There have been temptations of defining analytic instantaneous frequency based on this concept. Any new clarification and development in relation to phase derivative and analytic instantaneous frequency would lead to deeper understanding to the subject.

Remark 8.2 The developed theory and results are valid for both complex- and real-valued signals. Since for real-valued signals there holds $\hat{s}(-\omega)=\overline{\hat{s}}(\omega)$, we always have $\langle\omega\rangle=0$ (see Example 4.3). For a real-valued signal the mean $\langle\omega\rangle$ is often replaced by $\langle\omega\rangle^{+}$that presents a meaningful mean of the Fourier frequencies. The latter corresponds to $s^{+}$in the Hardy-Sobolev spaces decomposition $s=s^{+}+s^{-}$. 
Remark 8.3 In the generalized function theory it is well known that existence of the strong derivative $\frac{d s}{d t}$ as a function in $L_{l o c}^{1}(\mathbb{R})$ implies existence of the weak derivative $\frac{d^{*} s}{d t}$. The converse also holds: if $s, \frac{d^{*} s}{d t} \in L^{2}(\mathbb{R})$, then there exists an absolutely continuous function $\tilde{s}$ such that $s(t)=\tilde{s}(t)$ a.e., and $\frac{d \tilde{s}}{d t}=\frac{d^{*} s}{d t}([16])$. Therefore, the absolutely continuous function $\tilde{s}(t)$ is a representative of the Lebesgue equivalent class of $s$ in the Sobolev space. There is then the question whether we can use such an absolutely continuous function as an representative for the equivalent class $s$ for which $\frac{d^{*} s}{d t} \in L^{2}(\mathbb{R})$ ? The answer is "no", for a signal analysis theory should be based on the equivalent classes but not on particular representations. The amplitude derivatives and phase derivatives above defined through the Hardy-Sobolev spaces decomposition are not dependent of any particular representation $s$ but on the equivalent class $s$. Indeed, the analytic functions $s^{ \pm}$defined through formulas (2.16) do not vary if the boundary function $s$ is replaced by any function in the same equivalent class.

\section{References}

[1] H. Akçay, B. Niness: Orthonormal basis functions for modelling continuous-time systems. Signal Processing. 77, 261-274(1999)

[2] P. Auscher, E. Russ, Ph. Tchamitchian: Hardy Sobolev spaces on strong Lipchitz domains in $\mathbb{R}^{n}$. J. Funct. Anal. 218, 54-109(2005)

[3] A. Bultheel, P. González-Vera, E. Hendriksen, O.Njastad: Orthogonal Rational Functions, volume 5 of Cambridge Monographs on Applied and Computational Mathematics. Cambridge University Press(1999)

[4] L. Cohen: Time-Frequency Analysis: Theory and Applications. Prentice Hall(1995)

[5] L. Cohen, C. Lee: Instantaneous frequency, its standard deviation and multicomponent siganls. Proc. SPIE. 975, 186-208(1988)

[6] L. Cohen, C. Lee: Instantaneous bandwidth. In: B.Boashash(ed.) Time-Frequency Signal Analysis, pp.98-117. Longman Cheshire(1992)

[7] D. Gabor: Theory of communication. J.IEE. 93(III), 429-457(1946)

[8] J.B. Garnett: Bounded Analytic Functions. Academic Press(1987)

[9] P.S.C. Heuberger, P.M.J. Van den Hof, B. Wahlberg: Modelling and Identification with Rational Orthogonal Basis Functions. Springer Verlag(2005)

[10] N.E. Huang et al: The empirical mode decomposition and the Hilbert spectrum for nonlinear and non-stationary time series analysis. Proc. R. Soc. London A. 454, 903-995(1998)

[11] Z. Lou, A. McIntosh: Hardy spaces of exact form on $\mathbb{R}^{n}$. Trans. Amer. Math. Soc. 357, 14691496(2005)

[12] A.V. Oppenheim, R.W. Schafer: Discrete-Time Signal Processing. Prentice-Hall, Inc(1989)

[13] A.V. Oppenheim, A.S. Willsky, I.T. Young: Signals and Systems. Prentice-Hall, Inc(1983) 
[14] B. Picinbono: On instantaneous amplitude and phase of signals. IEEE Transactions on Signal Processing. 45, No. 3, March, 552-560(1997)

[15] Ch. Pommerrenke: Boundary Behaviour of Conformal Maps. Grundlehren der Mathematischen Wissenschaften. 299. Berlin: Springer- Verlag(1992)

[16] W.P. Ziemer: Weakly Differentiable Functions. Springer-Verlag New York Inc(1989)

[17] T. Qian: Mono-components for decomposition of signals. Math. Meth. Appl. Sci. 29, 1187$1198(2006)$

[18] T. Qian: Phase derivative of Nevanlinna functions and applications. Math. Meth. Appl. Sci. 32, 253-263(2009)

[19] T. Qian: Intrinsic mono-component decomposition of functions: an advance of Fourier theory. Math. Meth. Appl. Sci. DOI: 10.1002/mma.1214

[20] T. Qian, Y-B. Wang, P. Dang: Adaptive Decomposition Into Mono-Components. Advances in Adaptive Data Analysis. DOI No: 10.1142/S1793536909000278.

[21] W. Rudin: Real and Complex Analysis. New York, McGraw Hill(1966)

[22] R.C. Sharpley, V. Vatchev: Analysis of intrinsic mode functions. Constr. Approx. 24, 17-47(2006)

[23] E.M. Stein: Singular Integrals and Differentiability Properties of Functions. Princeton University Press, Princeton, New Jersey(1970) 